\title{
HAMILTONIAN STABILITY OF CERTAIN MINIMAL LAGRANGIAN SUBMANIFOLDS IN COMPLEX PROJECTIVE SPACES
}

\author{
Dedicated to Professor Koichi Ogiue on his sixtieth birthday
}

Amartuvshin Amarzaya AND YoshiHiro OHNITA

(Received February 20, 2002, revised November 15, 2002)

\begin{abstract}
A compact minimal Lagrangian submanifold immersed in a Kähler manifold is called Hamiltonian stable if the second variation of its volume is nonnegative under all Hamiltonian deformations. We study compact Hamiltonian stable minimal Lagrangian submanifolds with parallel second fundamental form embedded in complex projective spaces. Moreover, we completely determine Hamiltonian stability of all real forms in compact irreducible Hermitian symmetric spaces, which were classified previously by M. Takeuchi.
\end{abstract}

Introduction. Let $(M, \omega)$ be a $2 n$-dimensional symplectic manifold with a symplectic form $\omega$ and $L$ be an $n$-dimensional smooth manifold. An immersion $\varphi: L \rightarrow M$ is called a Lagrangian immersion if the 2-form $\varphi^{*} \omega$ on $L$ pulled back by the immersion $\varphi: L \rightarrow M$ vanishes identically. Then $L$ is called a Lagrangian submanifold immersed in a symplectic manifold $M$.

We say that a compact Lagrangian submanifold immersed in a Kähler manifold $M$ is an $H$-minimal Lagrangian submanifold if it has extremal volume under all Hamilitonian variations of the Lagrangian immersion. If a compact Lagrangian submanifold $L$ immersed in a Kähler manifold $M$ is minimal, in the sense that it has extremal volume under all smooth variations of the immersion, then it is always H-minimal. A compact H-minimal Lagrangian submanifold in a Kähler manifold $M$ is called Hamiltonian stable if the second variation for the volume is nonnegative for all Hamiltonian deformations of the Lagrangian immersion. Any compact stable, in the sense that the second variations are nonnegative under all smooth variations of the immersion, minimal submanifold $L$ immersed in a Kähler manifold $M$ is always H-stable.

In [18], [19], [20], [21], Oh developed the fundamental theory for Hamiltonian stability of H-minimal Lagrangian submanifolds of Kähler manifolds. It is known that every compact minimal Lagrangian submanifold $L$ in an Einstein-Kähler manifold $M$ with nonpositive Ricci curvature is stable ([5], [18]).

2000 Mathematics Subject Classification Primary: 53C42; Secondary： 53C40, 58G25.

Key words and phrases. Lagrangian submanifold, minimal submanifold, Hamiltonian stability, symplectic geometry. 
It is an interesting problem to classify all compact Hamiltonian stable minimal Lagrangian submanifolds $L$ in an Einstein-Kähler manifold $M$ with positive Ricci curvature. In this paper we study this problem in the case when $M$ is either a complex projective space or a compact Hermitian symmetric space. Compact stable minimal submanifolds in compact rank one symmetric spaces have already been classified by Lawson-Simons in [9] and the second named author in [22].

Let $\boldsymbol{C} P^{n}=\boldsymbol{C} P^{n}(c)$ be an $n$-dimensional complex projective space equipped with the Fubini-Study metric of constant holomorphic sectional curvature $c$. Let $S^{m}(c)$ denote an $m$ dimensional standard sphere of constant sectional curvature $c$ or radius $1 / \sqrt{c}$. The real projective subspace $\boldsymbol{R} P^{n} \subset \boldsymbol{C} P^{n}$ is the first example of a compact minimal Lagrangian submanifold embedded in $\boldsymbol{C} P^{n}$. Consider an $(n+1)$-dimensional torus $T^{n+1}=S^{1}((n+1) c / 4) \times$ $\cdots \times S^{1}((n+1) c / 4) \subset S^{2 n+1}(c / 4)$ naturally embedded in $C^{n+1}$. By the Hopf fibration $\pi: S^{2 n+1}(c / 4) \rightarrow C P^{n}$, we have the so-called Clifford torus $T^{n}=\pi\left(T^{n+1}\right) \subset C P^{n}$ of $C P^{n}$, which is the second example of a compact minimal Lagrangian submanifold embedded in $\boldsymbol{C} P^{n}$. Oh showed that the real projective subspaces $\boldsymbol{R} P^{n}$ and the Clifford tori $T^{n}$ are Hamiltonian stable minimal Lagrangian submanifolds embedded in $C P^{n}$ ([18]). Then the following is a natural and interesting problem (cf. [4]).

Problem. Determine all compact Hamiltonian stable minimal Lagrangian submanifolds in $\boldsymbol{C} P^{n}$.

The purpose of this paper is to study this problem for a class of compact Hamiltonian stable minimal Lagrangian submanifolds embedded in $C P^{n}$, including real projective subspaces $\boldsymbol{R} P^{n}$ and the Clifford tori $T^{n}$. Our main result is

THEOREM. Let $L$ be an $n$-dimensional compact totally real minimal submanifold embedded in $\boldsymbol{C P}^{n}$ with parallel second fundamental form in the following list:

(1) $S U(p) / Z_{p}, n=p^{2}-1$.

(2) $S U(p) / S O(p) Z_{p}, n=(p-1)(p+2) / 2$.

(3) $S U(2 p) / S p(p) Z_{2 p}, n=(p-1)(2 p+1)$.

(4) $E_{6} / F_{4} Z_{3}, n=26$.

Then $L$ is a Hamiltonian stable minimal Lagrangian submanifold in $\boldsymbol{C P}^{n}$.

In Section 1, we recall fundamental results on Hamiltonian stability of minimal Lagrangian submanifolds in Kähler manifolds. Section 2 is devoted to reviewing the classification theory of totally real submanifolds in $\boldsymbol{C} P^{n}$ with parallel second fundamental form. The proof of our main theorem is given in Sections 3 and 4. Finally, we study Hamiltonian stability of totally geodesic Lagrangian submanifolds in compact Hermitian symmetric spaces of rank greater than 1 in Section 5.

The authors would like to thank the referee for his careful reading of the manuscript.

1. Hamiltonian stability of minimal Lagrangian submanifolds in Kähler manifolds. Let $M$ be a $2 n$-dimensional symplectic manifold with symplectic form $\omega$, and let 
$L$ be an $n$-dimensional smooth submanifold of $M$. Let $\varphi: L \rightarrow M$ be a Lagrangian immersion. Set $N L:=\varphi^{-1} T M / \varphi_{*} T L$, the quotient vector bundle of $\varphi^{-1} T M$ by the subbundle $\varphi_{*} T L$. For each $v \in\left(\varphi^{-1} T M\right)_{x}$, define $\alpha_{v} \in T_{x}^{*} L$ by $\alpha_{v}(X):=\omega_{\varphi(x)}(v, X)$ for $X \in T_{x} L$. By the nondegeneracy of $\omega$ it induces a linear isomorphism $\varpi: N_{x} L \rightarrow T_{x}^{*} L$ by $v \mapsto \alpha_{v}$ and thus a bundle isomorphism $N L \rightarrow T^{*} L$.

By the definition a Lagrangian deformation of $\varphi$ is a smooth family $\left\{\varphi_{t}|| t \mid<\varepsilon\right\}$ of Lagrangian immersions of $L$ into $M$ with $\varphi_{0}=\varphi$. Such a deformation is characterized by a property that $\alpha_{V_{t}} \in \Omega^{1}(L)$ is closed for each $t$, where $V_{t}=\partial \varphi_{t} / \partial t$ is an infinitesimal deformation of $\left\{\varphi_{t}\right\}$ at a time $t$. An infinitesimal deformation $V \in C^{\infty}\left(\varphi^{-1} T M\right)$ is called Lagrangian if $\alpha_{V} \in \Omega^{1}(L)$ is closed and Hamiltonian if $\alpha_{V} \in \Omega^{1}(L)$ is exact. A smooth family $\left\{\varphi_{t}\right\}$ of Lagrangian immersions of $L$ into $M$ is called a Hamiltonian deformation of $\varphi=\varphi_{0}$ if its infinitesimal deformation $\partial \varphi_{t} / \partial t$ is Hamiltonian for each $t$.

Assume that $M$ is an $n$-dimensional Kähler manifold with complex structure $J$ and Kähler metric $g$. The Kähler form $\omega$ of $M$, which is defined by $\omega(X, Y):=g(J X, Y)$, defines a symplectic structure of $M$. If an immersion $\varphi: L \rightarrow M$ satisfies $J_{x}\left(\varphi_{*} T_{x} L\right) \subset T_{x}^{\perp} L$ for each $x \in L$, then $L$ is called a totally real submanifold immersed in a Kähler manifold $M$ ([6]). Here for $x \in L$ we define an orthogonal decomposition $T_{\varphi(x)} M=\varphi_{*} T_{x} L \oplus T_{x}^{\perp} L$ along $\varphi$ with respect to the metric $g$. We can identify the bundle $N L$ with the bundle $T^{\perp} L$. Then the complex structure $J$ induces a bundle isomorphism $N L \rightarrow \varphi_{*} T L$ preserving metrics and connections. Since we have $\alpha_{v}(X)=g_{\varphi(x)}\left(J v, \varphi_{*} X\right)$ for $X \in T_{x} L, \alpha_{v}$ corresponds to $J v$ through the linear isomorphism $T_{x}^{*} L \cong T_{x} L \cong \varphi_{*} T_{x} L$ with respect to $g$. Then we have a linear isomorphism

$$
\varpi: C^{\infty}\left(T^{\perp} L\right) \ni V \mapsto \alpha_{V} \in \Omega^{1}(L) .
$$

DEFINITION 1.1. A compact Lagrangian submanifold $L$ immersed in a Kähler manifold $M$ is called Hamiltonian minimal or H-minimal if the first variation for the volume of $L$ vanishes under all Hamiltonian deformations of $L$ in $M$.

Let $H$ denote the mean curvature vector field of a Lagrangian immersion $\varphi: L \rightarrow M$. If $M$ is an Einstein-Kähler manifold, then it satisfies $d \alpha_{H}=0$, that is, $\alpha_{H}$ is a closed 1-form on $L$ ([7]). In [20] it was shown that a Lagrangian submanifold $L$ immersed in a Kähler manifold $M$ is H-minimal if and only if $\delta \alpha_{H}=0$, where $\delta$ is the codifferential operator of $d$ with respect to the induced metric on $L$. If a Lagrangian immersion $\varphi: L \rightarrow M$ has parallel mean curvature vector field $H$ with respect to the normal connection, then it is H-minimal. Note that if $L$ is a compact H-minimal Lagrangian submanifold in an Einstein-Kähler manifold $M$ with $H^{1}(L, \boldsymbol{R})=\{0\}$ or more strongly with positive Ricci curvature, then the mean curvature vector field $H$ of $L$ vanishes, that is, $L$ is minimal.

Definition 1.2. A compact H-minimal Lagrangian submanifold $M$ immersed in a Kähler manifold is called Hamiltonian stable if the second variation for the volume of $M$ is nonnegative under all Hamiltonian deformations of $M$. 
Suppose that $L$ is a compact minimal Lagrangian submanifold immersed in an EinsteinKähler manifold $M$ with Einstein constant $\kappa$. Under the correspondence between $C^{\infty}(N L)$ and $\Omega^{1}(L)=d\left(\Omega^{0}(L)\right) \oplus \operatorname{Ker}\left(d^{*} \mid \Omega^{1}(L)\right)$, the Jacobi operator $\mathcal{J}$ of $L$ as a minimal submanifold corresponds to the linear operator $\tilde{\mathcal{J}}=\Delta^{1}-\kappa$ Id, where $\Delta^{1}=d \delta+\delta d$ denotes the Laplacian of $L$ acting on 1 -forms and Id denotes the identity operator. The second variation of the volume for a compact minimal Lagrangian submanifold under Hamiltonian deformations is described by the restriction of $\tilde{\mathcal{J}}$ to $d\left(\Omega^{0}(L)\right)$. The null space of $\mathcal{J}$ on Hamiltonian deformations corresponds to the null space of $\tilde{\mathcal{J}}$ on $d\left(\Omega^{0}(L)\right)$, and it is linearly isomorphic to the eigenspace of the Laplacian on $C^{\infty}(L)$ with eigenvalue $\kappa$. We denote by $n(L)$ the nullity of $L$, that is, the dimension of the null space.

Let $\mathcal{K}$ denote the space of Killing vector fields on a compact Einstein-Kähler manifold $M$ with positive Einstein constant $\kappa$. Assume that $\mathcal{K} \neq\{0\}$, or equivalently by a theorem of Lichnerowicz [10] and Matsushima [12], the first eigenvalue of the Laplacian acting on $C^{\infty}(M)$ is equal to $2 \kappa$. We denote by $\mathrm{V}_{1}(M)$ the corresponding eigenspace. By a theorem of Matsushima [12], we know that

$$
\mathcal{K}=\left\{J \operatorname{grad} f \in C^{\infty}(T M) \mid f \in \mathrm{V}_{1}(M)\right\} .
$$

For each $W \in \mathcal{K}$, we have an orthogonal decomposition $W=W^{T}+W^{\perp}$, where $W^{T}$ and $W^{\perp}$ denote the tangential and the normal components of the restriction of $W$ to $L$ in $M$. Set

$$
\mathcal{K}^{\perp}=\left\{W^{\perp} \in C^{\infty}(N L) \mid W \in \mathcal{K}\right\} .
$$

Then we have a linear isomorphism

$$
\mathcal{K}^{\perp} \cong \mathcal{K} /\left\{W \in \mathcal{K} \mid W^{\perp}=0\right\} .
$$

If $W=-J \operatorname{grad} f \in \mathcal{K}$ for the first eigenfunction $f$ of the Laplacian acting on $C^{\infty}(M)$, then it is easy to check that the formula

$$
d\left(\left.f\right|_{L}\right)=\alpha_{W^{\perp}}
$$

holds on $L$, which means that each $W^{\perp} \in \mathcal{K}^{\perp}$ is an infinitesimal Hamiltonian deformation. Hence, for a suitable constant $\alpha,\left.f\right|_{L}+\alpha$ is an eigenfunction of the Laplacian acting on $C^{\infty}(L)$ with eigenvalue $\kappa$. Set $n_{\mathcal{K}}(L)=\operatorname{dim} \mathcal{K}^{\perp}$. Since each $W \in \mathcal{K}$ with $W^{\perp}=0$ induces a Killing vector field on $L$, we obtain inequalities

$$
n(L) \geq n_{\mathcal{K}}(L) \geq \operatorname{dim} \mathcal{K}-\operatorname{dim} I_{0}(L),
$$

where $I_{0}(L)$ denotes the identity component of the isometry group of $L$. In the case when $M=C P^{n}$, since $\operatorname{dim} \mathcal{K}=\operatorname{dim} S U(n+1)=(n+1)^{2}-1$ and $\operatorname{dim} I_{0}(L) \leq n(n+1) / 2$, compact minimal Lagrangian submanifolds $L$ in $C P^{n}$ satisfy

$$
n(L) \geq n_{\mathcal{K}}(L) \geq \operatorname{dim} \mathcal{K}-\operatorname{dim} I_{0}(L) \geq n(n+3) / 2 .
$$

The Hamiltonian stability problem of compact minimal Lagrangian submanifolds in an Einstein-Kähler manifold is reduced to the first positive eigenvalue problem of the Laplacian acting on functions. 
THEOREM 1.1 ([18]). Let $M$ be an Einstein-Kähler manifold with Einstein constant $\kappa$. A compact minimal Lagrangian submanifold $L$ in $M$ is Hamiltonian stable if and only if $\lambda_{1} \geq \kappa$, where $\lambda_{1}>0$ is the first eigenvalue of the Laplacian acting on $C^{\infty}(L)$.

It is an important property of compact minimal Lagrangian submanifolds in $\boldsymbol{C} P^{n}(c)$ that if $f$ is the first eigenfunction of the Laplacian on $C P^{n}(c)$, then the restriction $\left.f\right|_{L}$ of $f$ to $L$ is the eigenfunction of the Laplacian on $L$ with eigenvalue $c(n+1) / 2$. This was used by Urbano [28] and a similar result was studied by Ono [23] for a class of generalized flag manifolds including Hermitian symmetric spaces.

THEOREM 1.2 ([28], [23]). Assume that $M$ is a compact Hermitian symmetric space with standard Einstein-Kähler metric and Einstein constant $\kappa$. Then a compact minimal Lagrangian submanifold $L$ in $M$ is Hamiltonian stable if and only if $\lambda_{1}=\kappa$. Here $\lambda_{1}$ is the first eigenvalue of the Laplacian acting on $C^{\infty}(L)$. In particular, when $M$ is a complex projective space $C P^{n}(c)$ with constant holomorphic sectional curvature $c$, a compact minimal Lagrangian submanifold $L$ in $C P^{n}(c)$ is Hamiltonian stable if and only if $\lambda_{1}=c(n+1) / 2$.

There is some topological restriction for compact minimal Lagrangian submanifolds in $C P^{n}$ to be Hamiltonian stable.

THEOREM 1.3. Let $L$ be a compact minimal Lagrangian submanifold immersed in $C P^{n}$. If $L$ is Hamiltonian stable, then $H_{1}(L ; Z) \neq\{0\}$. In particular, L cannot be simply connected.

Proof. By $\varphi: L \rightarrow C P^{n}$ we denote the Lagrangian immersion and by $\pi: S^{2 n+1}(c /$ $4) \rightarrow C P^{n}(c)=C P^{n}$ the Hopf fibration. On the $S^{1}$-bundle $\varphi^{-1} S^{2 n+1} \rightarrow L$ pulled-back by $\varphi$ from the Hopf fibration, the induced connection is flat, by virtue of the Lagrangian property of $\varphi$. Let $\rho: \pi_{1}(L) \rightarrow S^{1}$ be the holonomy homomorphism of the flat connection. Since $\pi_{1}(L) / \operatorname{Ker} \rho \cong \operatorname{Im} \rho \subset S^{1}$ is abelian, the natural homomorphism $H_{1}(L ; Z) \cong$ $\pi_{1}(L) /\left[\pi_{1}(L), \pi_{1}(L)\right] \rightarrow \pi_{1}(L) / \operatorname{Ker} \rho$ is surjective. Assume that $H_{1}(L ; \boldsymbol{Z})=\{0\}$. Then $\rho$ is trivial. Hence there is a minimal isometric immersion $\tilde{\varphi}: L \rightarrow S^{2 n+1}(c / 4)$ which is horizontal with respect to $\pi: S^{2 n+1}(c / 4) \rightarrow C P^{n}$ such that $\varphi=\tilde{\varphi} \circ \pi$. By Takahashi's theorem [24], component functions of $\tilde{\varphi}: L \rightarrow S^{2 n+1}(c / 4)$ are eigenfunctions of the Laplaician of $L$ with eigenvalue $c n / 4$. Hence the first eigenvalue $\lambda_{1}$ of the Laplacian satisfies $\lambda_{1} \leq c n / 4<c(n+1) / 2$. Thus the minimal Lagrangian immersion $\varphi: L \rightarrow C P^{n}$ is not Hamiltonian stable.

REMARK 1. This result does not hold in the case of compact Hermitian symmetric space of rank greater than 1 (see Section 4).

2. Minimal Lagrangian submanifolds in $C P^{n}$ with parallel second fundamental form. Totally real submanifolds in a complex projective space have been much investigated after the publication of the paper [6] (cf. [5] and [17]). Also totally real submanifolds in complex space forms (that is, a complex Euclidean space $\boldsymbol{C}^{n}$, a complex projective space $\boldsymbol{C} \boldsymbol{P}^{n}$ 
and a complex hyperbolic space $\boldsymbol{C} H^{n}$ ) with parallel second fundamental form were elaborately classified by Naitoh and Naitoh-Takeuchi in [14], [15], [13], [16]. Since the parallelism of the second fundamental form implies the parallelism of the mean curvature vector field, such submanifolds provide a nice class of $\mathrm{H}$-minimal Lagrangian submanifolds in complex projective spaces.

By virtue of their theory, all totally real submanifolds in $C P^{n}$ with parallel second fundamental form are constructed in the following way. Let $(U, G)$ be a Hermitian symmetric pair of compact type with the canonical decomposition $\mathfrak{u}=\mathfrak{g}+\mathfrak{p}$. Set $\operatorname{dim}(U / G)=2(n+1)$. Let $\langle,\rangle_{\mathfrak{u}}$ denote the $\operatorname{Ad}(U)$-invariant inner product of $\mathfrak{u}$ defined by $(-1)$-times Killing-Cartan form of $\mathfrak{u}$. Relative to the complex structure the subspace $\mathfrak{p}$ can be identified with a complex Euclidean space $\boldsymbol{C}^{n+1}$. We take the decomposition of $(U, G)$ into irreducible Hermitian symmetric pairs of compact type:

$$
(U, G)=\left(U_{1}, G_{1}\right) \oplus \cdots \oplus\left(U_{s}, G_{s}\right) .
$$

$\operatorname{Set} \operatorname{dim}\left(U_{i} / G_{i}\right)=2\left(n_{i}+1\right)$ for $i=1, \ldots, s$. Let $\mathfrak{u}_{i}=\mathfrak{g}_{i}+\mathfrak{p}_{i}$ be the canonical decomposition of $\left(U_{i}, G_{i}\right)$ for each $i=1,2, \ldots, s$. Assume that there is an element $\eta_{i} \in \mathfrak{p}_{i}$ satisfying the condition $\left(\operatorname{ad} \eta_{i}\right)^{3}+4\left(\operatorname{ad} \eta_{i}\right)=0$. Choose positive numbers $c_{1}>0, \ldots, c_{s}>0$ with $\sum_{i=1}^{s} 1 / c_{i}=1 / c$. Put $a_{i}=1 / \sqrt{2 c_{i}\left(n_{i}+1\right)}$ for each $i=1, \ldots, s$. Set $\hat{L}_{i}=$ $\operatorname{Ad}\left(G_{i}\right)\left(a_{i} \eta_{i}\right) \subset S^{2 n_{i}+1}\left(c_{i} / 4\right) \subset \mathfrak{p}_{i}$. It should be remarked that $\hat{L}_{i}$ is an irreducible symmetric $R$-space standardly embedded in a complex Euclidean space $\mathfrak{p}_{i}$.

Let $\eta=a_{1} \eta_{1}+\cdots+a_{s} \eta_{s} \in \mathfrak{p}$. Let $\hat{L}=\operatorname{Ad}(G)(\eta) \subset S^{2 n+1}(c / 4) \subset \mathfrak{p}$, which is a symmetric $R$-space standardly embedded in a complex Euclidean space $\mathfrak{p}$. Then we have the inclusions:

$$
\hat{L}=\hat{L}_{1} \times \cdots \times \hat{L}_{s} \subset S^{2 n_{1}+1}\left(c_{1} / 4\right) \times \cdots \times S^{2 n_{s}+1}\left(c_{s} / 4\right) \subset S^{2 n+1}(c / 4) .
$$

THEOREM 2.1 ([16]). Let $\pi: S^{2 n+1}(c / 4) \rightarrow C P^{n}(c)$ be the Hopf fibration and put $L=\pi(\hat{L})$. Then

(1) L is a compact totally real submanifold embedded in $C P^{n}(c)$ with parallel second fundamental form, and thus $L$ is a symmetric space.

(2) L is a minimal submanifold in $\boldsymbol{C} P^{n}(c)$ if and only if $c_{i}\left(n_{i}+1\right)=c(n+1)$ for each $i=1, \ldots, s$.

(3) The dimension of the Euclidean factor of $L$ is equal to $s-1$.

(4) $L$ is flat if and only if $s=n+1$.

(5) L has no Euclidean factor if and only if $s=1$. In this case $L$ is an irreducible symmetric space and a minimal submanifold in $\boldsymbol{C} P^{n}$.

(6) Conversely every totally real submanifold immersed in $\boldsymbol{C P}^{n}$ with parallel second fundamental form covers an open submanfold of $L$ constructed in this way.

Note that $L$ is always a compact H-minimal Lagrangian submanifold embedded in $C P^{n}(c)$. Note that $\hat{L}$ is also a compact H-minimal Lagrangian submanifold in $\boldsymbol{C}^{n+1}$. Their Hamiltonian stability will be discussed in a forthcoming paper [2]. 
In the case when $L$ is flat, $L$ is the Clifford torus of $\boldsymbol{C} P^{n}$. In the case when $L$ has no Euclidean factor, $L$ is locally isometric to one of the following symmetric spaces: $S^{n}(c / 4)$, $S U(p), S U(p) / S O(p), S U(2 p) / S p(p), E_{6} / F_{4}$. Their explicit descriptions are given in Section 5 of [13]. From now on we only discuss this case.

Suppose that $(U, G)$ is irreducible $(s=1)$. In order to determine the Hamiltonian stability of the Lagrangian submanifold $L$ embedded in $\boldsymbol{C} P^{n}$, we have to calculate the first eigenvalue $\lambda_{1}$ of the Laplacian on $C^{\infty}(L)$ with respect to the induced metric on $L$ from the Fubini-Study metric of $\boldsymbol{C} P^{n}$.

Let $\widetilde{L}$ denote the universal covering space of $L$. Let $\tilde{\lambda}_{1}$ denote the first eigenvalue of the Laplacian acting on $C^{\infty}(\widetilde{L})$. The complete list of all irreducible Hermitian symmetric spaces $U / G$ of compact type and corresponding $L, \widetilde{L}$ is given as follows:

\begin{tabular}{|c|c|c|c|c|}
\hline Class & $U / G$ & $n+1$ & $L$ & $\widetilde{L}$ \\
\hline AIII & $\frac{S U(2 p)}{S(U(p) \times U(p))}$ & $p^{2}$ & $S U(p) \boldsymbol{Z}_{p}$ & $S U(p)$ \\
\hline DIII & $\frac{S O(4 p)}{U(2 p)}$ & $p(2 p-1)$ & $\frac{S U(2 p)}{S p(p) \boldsymbol{Z}_{2 p}}$ & $\frac{S U(2 p)}{S p(p)}$ \\
\hline BDI & $\frac{S O(p+2)}{S O(p) \times S O(2)}$ & $p$ & $\boldsymbol{R} P^{n}$ & $S^{n}$ \\
\hline CI & $\frac{S p(p)}{U(p)}$ & $\frac{p(p+1)}{2}$ & $\frac{S U(p)}{S O(p) Z_{p}}$ & $\frac{S U(p)}{S O(p)}$ \\
\hline EVII & $\frac{E_{7}}{E_{6} \cdot T}$ & 27 & $\frac{E_{6}}{F_{4} \boldsymbol{Z}_{3}}$ & $\frac{E_{6}}{F_{4}}$ \\
\hline
\end{tabular}

Here note that the compact symmetric space $L$ is a bottom space.

We take the decomposition

$$
\mathfrak{g}=\mathfrak{c}(\mathfrak{g}) \oplus \mathfrak{g}^{\prime}
$$

into the center and the semisimple part of $\mathfrak{g}$.

Let us denote by $B_{\mathfrak{h}}($, ) the Killing-Cartan form of a Lie algebra $\mathfrak{h}$. Define the invariant inner products on $\mathfrak{u}$ and $\mathfrak{g}^{\prime}$ by

$$
\langle,\rangle_{\mathfrak{u}}:=-B_{\mathfrak{u}}(,)
$$

and

$$
\langle,\rangle_{\mathfrak{g}^{\prime}}:=-B_{\mathfrak{g}^{\prime}}(,),
$$

respectively. For $\eta \in \mathfrak{p}$ with $\langle\eta, \eta\rangle_{\mathfrak{u}}=4 / c$, put

$$
\mathfrak{k}:=\left\{X \in \mathfrak{g}^{\prime} \mid[X, \eta]=0\right\} .
$$


Then we have an orthogonal decomposition

$$
\mathfrak{g}^{\prime}=\mathfrak{k}+\mathfrak{m}
$$

Define an inner product on $\mathfrak{m}$ by

$$
\langle X, Y\rangle:=\langle[X, \eta],[Y, \eta]\rangle_{\mathfrak{u}}
$$

for $X, Y \in \mathfrak{m}$, which corresponds to the induced metric on $L$ from the Fubini-Study metric of $C P^{n}$.

Now we assume that there exists a constant $C>0$ such that

$$
\langle X, Y\rangle=C\langle X, Y\rangle_{\mathfrak{g}^{\prime}}
$$

for $X, Y \in \mathfrak{m}$. Then the constant $C>0$ can be given explicitly as follows.

LEMMA 2.1.

$$
C=\frac{2}{c}\left(\frac{1}{n+1}-\frac{2}{\operatorname{dimg}^{\prime}} a(\mathfrak{p})\right),
$$

where $a(\mathfrak{p})$ denotes the eigenvalue of the Casimir operator on the representation space $\mathfrak{p}$ of $\mathfrak{g}^{\prime}$ with respect to $\langle,\rangle_{\mathfrak{g}^{\prime}}$.

This lemma follows immediately from Propositions 2.1 and 2.2.

Assume that there is a constant $l>0$ such that

$$
\langle X, Y\rangle_{\mathfrak{u}}=l\langle X, Y\rangle_{\mathfrak{g}^{\prime}}
$$

for $X, Y \in \mathfrak{g}^{\prime}$.

Set $m=\operatorname{dim} \mathfrak{m}$. Then we obtain the following formula for $C$ in terms of $l$.

Proposition 2.1. Let $\langle$,$\rangle be the inner product of \mathfrak{m}$ defined in (2.8). Assume that

$$
\langle X, Y\rangle=C\langle X, Y\rangle_{\mathfrak{g}^{\prime}}
$$

for $X, Y \in \mathfrak{m}$. Then we have

$$
C=\frac{l}{m} \frac{2 n}{c(n+1)}
$$

ProOF. Let $\left\{e_{i}\right\}$ be an orthonormal basis of $\mathfrak{m}$ with respect to $\langle,\rangle_{\mathfrak{u}}$. By using (2.9) and (2.11), we have

$$
\begin{aligned}
\sum_{i=1}^{m}\left\langle e_{i}, e_{i}\right\rangle & =C \sum_{i=1}^{m}\left\langle e_{i}, e_{i}\right\rangle_{\mathfrak{g}^{\prime}}=\frac{C}{l} \sum_{i=1}^{m}\left\langle e_{i}, e_{i}\right\rangle_{\mathfrak{u}} \\
& =\frac{C}{l} \operatorname{dim} \mathfrak{m}=\frac{C}{l} m .
\end{aligned}
$$


On the other hand, we obtain

$$
\begin{aligned}
\sum_{i=1}^{m}\left\langle e_{i}, e_{i}\right\rangle & =\sum_{i=1}^{m}\left\langle\left[e_{i}, \eta\right],\left[e_{i}, \eta\right]\right\rangle_{\mathfrak{u}} \\
& =-\sum_{i=1}^{m}\left\langle(\operatorname{ad} \eta)^{2} e_{i}, e_{i}\right\rangle_{\mathfrak{u}}=-\operatorname{tr}_{\mathfrak{g}^{\prime}}(\operatorname{ad} \eta)^{2} \\
& =-\left(\operatorname{tr}_{\mathfrak{g}}(\operatorname{ad} \eta)^{2}-\operatorname{tr}_{\mathfrak{c}_{\mathfrak{g}}}(\operatorname{ad} \eta)^{2}\right) \\
& =-\frac{1}{2} \operatorname{tr}_{\mathfrak{u}}(\operatorname{ad} \eta)^{2}+\operatorname{tr}_{\mathfrak{c}_{\mathfrak{g}}}(\operatorname{ad} \eta)^{2} \\
& =\frac{1}{2}\langle\eta, \eta\rangle_{\mathfrak{u}}-\frac{1}{2(n+1)}\langle\eta, \eta\rangle_{\mathfrak{u}} \\
& =\frac{1}{2} \frac{4}{c}-\frac{1}{2(n+1)} \frac{4}{c}=\frac{2 n}{c(n+1)}
\end{aligned}
$$

Next we are going to prove a formula determining the positive constant $l$. Here we can show a similar formula in a slightly general setting. Let $(\mathfrak{u}, \mathfrak{g})$ be an irreducible symmetric Lie algebra of compact type with canonical decomposition $\mathfrak{u}=\mathfrak{g}+\mathfrak{p}$. Assume that $\mathfrak{g}=$ $\mathfrak{g}_{0}^{\prime} \oplus \mathfrak{g}_{1}^{\prime} \oplus \cdots \oplus \mathfrak{g}_{t}^{\prime}$ is a decomposition into the center and simple ideals, where $\mathfrak{g}_{0}^{\prime}=\mathfrak{c}(\mathfrak{g})$.

PROPOSITION 2.2. If there is a constant $l_{1}>0$ such that

$$
\langle X, Y\rangle_{\mathfrak{u}}=l_{1}\langle X, Y\rangle_{\mathfrak{g}_{1}^{\prime}}
$$

for $X, Y \in \mathfrak{g}_{1}^{\prime}$, where

$$
\langle,\rangle_{\mathfrak{g}_{1}^{\prime}}=-B_{\mathfrak{g}_{1}^{\prime}}(,) .
$$

Then the constant $l_{1}>0$ is given by

$$
l_{1}=\frac{1}{k_{1}}\left(k_{1}-(\operatorname{dim} \mathfrak{p}) a^{\prime}(\mathfrak{p})\right),
$$

where $k_{1}=\operatorname{dim} \mathfrak{g}_{1}^{\prime}$ and $a^{\prime}(\mathfrak{p})$ denotes the eigenvalue of the Casimir operator on the representation space $\mathfrak{p}$ of $\mathfrak{g}_{1}^{\prime}$ with respect to $\langle,\rangle_{\mathfrak{g}_{1}^{\prime}}$. we have

PROOF. Let $\left\{X_{1}, \ldots, X_{k}\right\}$ be an orthonormal basis of $\mathfrak{g}_{1}^{\prime}$ with respect to $\langle,\rangle_{\mathfrak{g}_{1}^{\prime}}$. Then

$$
\sum_{j=1}^{k_{1}}\left\langle X_{j}, X_{j}\right\rangle_{\mathfrak{u}}=l_{1} \sum_{j=1}^{k_{1}}\left\langle X_{j}, X_{j}\right\rangle_{\mathfrak{g}_{1}^{\prime}}=l_{1} k_{1}
$$


On the other hand, we obtain

$$
\begin{aligned}
\sum_{j=1}^{k_{1}}\left\langle X_{j}, X_{j}\right\rangle_{\mathfrak{u}} & =-\sum_{j=1}^{k_{1}} \operatorname{tr}_{\mathfrak{u}}\left(\operatorname{ad} X_{j}\right)^{2} \\
& =-\sum_{j=1}^{k_{1}} \operatorname{tr}_{\mathfrak{g}}\left(\operatorname{ad} X_{j}\right)^{2}-\sum_{j=1}^{k_{1}} \operatorname{tr}_{\mathfrak{p}}\left(\operatorname{ad} X_{j}\right)^{2} \\
& =-\sum_{j=1}^{k_{1}} \operatorname{tr}_{\mathfrak{g}_{1}^{\prime}}\left(\operatorname{ad} X_{j}\right)^{2}-\sum_{j=1}^{k_{1}} \operatorname{tr}_{\mathfrak{p}}\left(\operatorname{ad} X_{j}\right)^{2} \\
& =\sum_{j=1}^{k_{1}}\left\langle X_{j}, X_{j}\right\rangle_{\mathfrak{g}_{1}^{\prime}}-\operatorname{tr}_{\mathfrak{p}}(\mathcal{C}) \\
& =k_{1}-(\operatorname{dim} \mathfrak{p}) a^{\prime}(\mathfrak{p}),
\end{aligned}
$$

where $\mathcal{C}=\mathcal{C}_{\left(\mathfrak{g}_{1}^{\prime},\langle,\rangle_{\mathfrak{g}_{1}^{\prime}}\right.}$ denotes the Casimir operator of $\mathfrak{g}_{1}^{\prime}$ with respect to $\langle,\rangle_{\mathfrak{g}_{1}^{\prime}}$.

3. Calculation of the first eigenvalues of minimal Lagrangian submanifolds in $C P^{n}$ with parallel second fundamental form. To calculate the eigenvalues of the Laplacian acting on functions we review relevant results from the theory of spherical functions on compact symmetric spaces ([26]). Let $G / K$ be a compact symmetric space with symmetric pair $(G, K, \sigma)$, where $G$ is a compact connected Lie group. Let $\mathfrak{g}=\mathfrak{k}+\mathfrak{m}$ be its canonical decomposition and $\mathfrak{a}$ be a maximal abelian subspace of $\mathfrak{m}$. We fix an $\operatorname{Ad} G$-invariant inner product ( , ) of $\mathfrak{g}$. Let $\mathfrak{t}$ be a maximal abelian subalgebra of $\mathfrak{g}$ containing $\mathfrak{a}$. Then we have $\mathfrak{t}=\mathfrak{b}+\mathfrak{a}$, where $\mathfrak{b}=\mathfrak{t} \cap \mathfrak{k}$. We fix a $\sigma$-linear order $<$ on $\mathfrak{t}$. The maximal torus $T$ of $G$ is generated by $\mathfrak{t}$. For each $\alpha \in \mathfrak{t}$, we put

$$
\tilde{\mathfrak{g}}_{\alpha}=\left\{X \in \mathfrak{g}^{C} \mid(\operatorname{ad} H) X=2 \pi \sqrt{-1}(\alpha, H) X \text { for each } H \in \mathfrak{t}\right\} .
$$

An element $\alpha \in \mathfrak{t}$ is called a root of $\mathfrak{g}$ with respect to $\mathfrak{t}$ if $\tilde{\mathfrak{g}}_{\alpha}$ is nonzero. We denote by $\Sigma(G)$ and $\Sigma^{+}(G)$ the set of all roots and all positive roots of $\mathfrak{g}$ with respect to $\mathfrak{t}$, respectively, and put $\Sigma_{0}(G)=\Sigma(G) \cap \mathfrak{b}$. Then we have the root decomposition

$$
\mathfrak{g}^{C}=\mathfrak{t}^{C}+\sum_{\alpha \in \Sigma(G)} \tilde{\mathfrak{g}}_{\alpha}
$$

We always denote by $\Pi(\mathfrak{g})$ the fundamental system of roots and set $\Pi_{0}(\mathfrak{g})=\Pi(\mathfrak{g}) \cap \Sigma_{0}(G)$. Set

$$
\begin{aligned}
& \Gamma(G):=\{H \in \mathfrak{t} \mid \exp H=e\}, \\
& Z(G):=\{\Lambda \in \mathfrak{t} \mid(\Lambda, H) \in \boldsymbol{Z} \text { for each } H \in \Gamma(G)\}, \\
& D(G):=\left\{\Lambda \in Z(G) \mid(\Lambda, \alpha) \geq 0 \text { for each } \alpha \in \Sigma^{+}(G)\right\} .
\end{aligned}
$$


Let $\mathcal{D}(G)$ be the complete set of inequivalent irreducible unitary representations of $G$. Then it is known that for each $(V, \rho) \in \mathcal{D}(G)$ the highest weight $\Lambda_{\rho}$ of $(V, \rho)$ belongs to $D(G)$, and the mapping $\mathcal{D}(G) \rightarrow D(G)$ is bijective.

Let $A$ be the torus of $G$ generated by $\mathfrak{a}$ and $\hat{A}=A o$ be a maximal torus of $G / K$, where $o$ denotes the origin $e K$ of $G / K$. For each $\gamma \in \mathfrak{a}$, we put

$$
\mathfrak{g}_{\gamma}=\left\{X \in \mathfrak{g}^{C} \mid(\operatorname{ad} H) X=2 \pi \sqrt{-1}(\alpha, H) X \text { for each } H \in \mathfrak{a}\right\} .
$$

An element $\gamma \in \mathfrak{a}$ is called a root of $\mathfrak{g}$ with respect to $\mathfrak{a}$ if $\mathfrak{g}_{\gamma}$ is nonzero. We denote by $\Sigma(G, K)$ and $\Sigma^{+}(G, K)$ the set of all roots and all positive roots of $\mathfrak{g}$ with respect to $\mathfrak{a}$, respectively. Then we have the decomposition

$$
\mathfrak{g}^{C}=\mathfrak{g}_{0}+\sum_{\gamma \in \Sigma(G, K)} \mathfrak{g}_{\gamma} .
$$

Put

$$
\begin{aligned}
\Gamma(G, K) & :=\{H \in \mathfrak{a} \mid(\exp H) o=o\}, \\
Z(G, K) & :=\{\Lambda \in \mathfrak{a} \mid(\Lambda, H) \in Z \text { for each } H \in \Gamma(G, K)\}, \\
D(G, K) & :=\left\{\Lambda \in Z(G, K) \mid(\Lambda, \gamma) \geq 0 \text { for each } \gamma \in \Sigma^{+}(G, K)\right\} .
\end{aligned}
$$

Then we have $Z(G, K) \subset Z(G)$ and $D(G, K) \subset D(G)$. Let $\mathcal{D}(G, K)$ be the complete set of inequivalent class-one unitary representations of pair $(G, K)$. Then for each $\left(V_{\rho}, \rho\right) \in$ $\mathcal{D}(G, K)$ the subspace $\left(V_{\rho}\right)_{K}=\left\{v \in V_{\rho} \mid \rho(k) v=v\right.$ for each $\left.k \in K\right\}$ is of complex dimension 1, and the bijection $\mathcal{D}(G) \rightarrow D(G)$ induces the bijection $\mathcal{D}(G, K) \rightarrow D(G, K)$.

Let $\Pi(G, K)=\left\{\gamma_{1}, \ldots, \gamma_{l}\right\}$ be the fundamental root system for $\Sigma(G, K)$. We define

$$
\beta_{i}= \begin{cases}\gamma_{i} & \text { if } 2 \gamma_{i} \notin \Sigma(G, K) \\ 2 \gamma_{i} & \text { if } 2 \gamma_{i} \in \Sigma(G, K),\end{cases}
$$

and set $\beta_{i}^{*}=2 \beta_{i} /\left(\beta_{i}, \beta_{i}\right)$. It is known (cf. [11]) that if $G / K$ is simply connected, then

$$
\Gamma(G, K)=\sum_{i=1}^{l} z\left(\frac{1}{2} \beta_{i}^{*}\right),
$$

and if $G / K$ is a bottom space, then

$$
\Gamma(G, K)=\left\{H \in \mathfrak{a} \mid\left(H, \frac{1}{2 \pi} \gamma_{i}\right) \in \frac{1}{2} Z, i=1,2, \ldots, p-1\right\} .
$$

Let $g$ be a $G$-invariant Riemannian metric on $G / K$ induced by (, ). If $G / K$ is irreducible and $g$ is a $G$-invariant Riemannian metric induced from the (-1)-times KillingCartan form, then $(G / K, g)$ is an Einstein manifold with Einstein constant $1 / 2$ (cf. [27]). Let $\Delta$ be the Laplacian of $(G / K, g)$ acting on functions, and let $\Lambda=\Lambda_{\rho}$ be the highest weight of the representation $\rho \in \mathcal{D}(G, K)$. Then by the Freudenthal formula the complete set of eigenvalues of $\Delta$ is given by

$$
\left\{-a_{\Lambda}=4 \pi^{2}\left(\Lambda_{\rho}+2 \delta(G), \Lambda_{\rho}\right) \mid \rho \in \mathcal{D}(G, K)\right\} .
$$


Here we set

$$
\delta(G)=\frac{1}{2} \sum_{\alpha \in \Sigma^{+}(G)} \alpha .
$$

The multiplicity of the $k$-th eigenvalue $\mu_{k}$ of $\Delta$ is given by $\sum_{\rho} d_{\rho}$, where the summation runs over all $\rho \in \mathcal{D}(G, K)$ such that $\mu_{k}=-a_{\Lambda}$. By Weyl's dimension formula $d_{\rho}$ is given by

$$
d_{\rho}=\operatorname{dim}\left(V_{\rho}, \rho\right)=\prod_{\alpha \in \Sigma^{+}(G)} \frac{\left(\alpha, \Lambda_{\rho}+\delta(G)\right)}{(\alpha, \delta(G))} .
$$

Let $\left\{\Lambda_{1}, \ldots, \Lambda_{l^{\prime}}\right\}$ be the fundamental weight system of the semisimple part $\mathfrak{g}^{\prime}$ of $\mathfrak{g}$, where $l^{\prime}=\operatorname{rank} \mathfrak{g}^{\prime}$. Then the highest weight $\Lambda(\mathfrak{p})$ of the isotropy representation of irreducible Hermitian symmetric pair $(U, G)$ restricted to $\mathfrak{g}^{\prime}$ and the corresponding eigenvalue $a(\mathfrak{p})$ of their Casimir operator with respect to the $(-1)$-times Killing-Cartan form of $\mathfrak{g}^{\prime}$ is given in the following list (cf. [8, Chapter 8]). Moreover, the constant $C$ in (2.9) is also given in each case.

\begin{tabular}{|c|c|c|c|c|c|c|}
\hline Class & $\mathfrak{g}^{\prime}$ & $\Lambda(\mathfrak{p})$ & $n+1$ & $\operatorname{dim} \mathfrak{g}^{\prime}$ & $-a(\mathfrak{p})$ & $C$ \\
\hline $\mathrm{AIII}$ & $\mathfrak{s u}(p) \oplus \mathfrak{s u}(p)$ & $\Lambda_{1} \otimes \Lambda_{p-1}$ & $p^{2}$ & $2\left(p^{2}-1\right)$ & $\frac{p^{2}-1}{p^{2}}$ & $\frac{4}{p^{2} c}$ \\
\hline $\mathrm{DIII}$ & $\mathfrak{s u}(2 p)$ & $\Lambda_{2}$ & $2 p^{2}-p$ & $4 p^{2}-1$ & $\frac{2 p^{2}-p-1}{p^{2}}$ & $\frac{2}{p^{2} c}$ \\
\hline $\mathrm{BI}$ & $\mathfrak{s o}(2 p-1)$ & $\Lambda_{1}$ & $2 p-1$ & $2 p^{2}-3 p+1$ & $\frac{p-1}{2 p-3}$ & $\frac{1}{(n-1) c}$ \\
\hline $\mathrm{DI}$ & $\mathfrak{s o}(2 p)$ & $\Lambda_{1}$ & $2 p$ & $p(2 p-1)$ & $\frac{2 p-1}{4(p-1)}$ & $\frac{1}{(n-1) c}$ \\
\hline $\mathrm{CI}$ & $\mathfrak{s u}(p)$ & $2 \Lambda_{1}$ & $\frac{p(p+1)}{2}$ & $p^{2}-1$ & $\frac{p^{2}+p-2}{p^{2}}$ & $\frac{8}{p^{2} c}$ \\
\hline $\mathrm{EVII}$ & $\mathfrak{e}_{6}$ & $\Lambda_{1}$ & 27 & 78 & $\frac{13}{18}$ & $\frac{1}{9 c}$ \\
\hline
\end{tabular}

Here we give a computation of $C$ only in the case of EVII. In this case $(U, G)=\left(E_{7}, T\right.$. $\left.E_{6}\right)$ and $(G, K)=\left(T \cdot E_{6}, F_{4}\right)$. From (2.12) and (2.16) we have

$$
C=\frac{2}{c}\left(\frac{1}{n+1}-\frac{2}{\operatorname{dimg}^{\prime}} a(\mathfrak{p})\right)=\frac{2}{c}\left(\frac{1}{27}+\frac{2}{78} \frac{13}{18}\right)=\frac{1}{9 c} \text {. }
$$

LEMma 3.1. Assume that $G / K$ is one of compact irreducible symmetric spaces $\boldsymbol{R} P^{n}=$ $S O(n+1) / S(O(n) \times O(1)), \quad S U(p) / Z_{p}, \quad S U(p) / S O(p) Z_{p}, \quad S U(2 p) / S p(p) Z_{2 p}$ and $E_{6} / F_{4} Z_{3}$. Then the first eigenvalue $\mu_{1}$ of the Laplacian for functions on $G / K$ with respect to the metric defined by the (-1)-times Killing-Cartan form of $\mathfrak{g}$ is given in the following list: 


\begin{tabular}{|c|c|c|c|c|}
\hline$G / K$ & $\widetilde{G / K}$ & $n=\operatorname{dim} G / K$ & $\mu_{1}$ & $\tilde{\mu}_{1}$ \\
\hline $\boldsymbol{R} P^{n}$ & $S^{n}$ & $n$ & $\frac{n+1}{2(n-1)}$ & $\frac{n}{4(n-1)}$ \\
\hline$\frac{S U(p)}{\boldsymbol{Z}_{p}}$ & $S U(p)$ & $p^{2}-1$ & 2 & $\frac{2\left(p^{2}-1\right)}{p^{2}}$ \\
\hline$\frac{S U(p)}{S O(p) \boldsymbol{Z}_{p}}$ & $\frac{S U(p)}{S O(p)}$ & $\frac{(p-1)(p+2)}{2}$ & $\frac{2(p+1)}{p}$ & $\frac{(p-1)(p+2)}{p^{2}}$ \\
\hline$\frac{S U(2 p)}{S p(p) \boldsymbol{Z}_{2 p}}$ & $\frac{S U(2 p)}{S p(p)}$ & $(p-1)(2 p+1)$ & $\frac{2 p-1}{p}$ & $\frac{(p-1)(2 p+1)}{2 p^{2}}$ \\
\hline$\frac{E_{6}}{F_{4} \boldsymbol{Z}_{3}}$ & $\frac{E_{6}}{F_{4}}$ & 26 & $\frac{3}{2}$ & $\frac{13}{18}$ \\
\hline
\end{tabular}

Here $\widetilde{G / K}$ is the universal covering space of $G / K, \tilde{\mu}_{1}$ denotes the first eigenvalue of the Laplacian acting on functions for $\widetilde{G / K}$ and $p \geq 2$ is an integer.

PRoof. We use the table of root systems for complex simple Lie algebras in [3].

The case $G / K=\left(S U(p) / Z_{p} \times S U(p) / Z_{p}\right) /\left(S U(p) / Z_{p}\right) \cong S U(p) / Z_{p}$ : Suppose that $\mathfrak{k}=\mathfrak{s u}(p)$ is a compact simple Lie algebra of type $A_{p-1}$. Let $\mathfrak{t}$ be a maximal abelian subalgebra of $\mathfrak{k}$. If $\left\{\varepsilon_{1}, \ldots, \varepsilon_{p}\right\}$ denotes the standard orthonormal basis of $\boldsymbol{R}^{p}$, then $\mathfrak{t}$ can be identified with the subspace of $\boldsymbol{R}^{p}$ as follows:

$$
\mathfrak{t}=\left\{\sum_{i=1}^{p} x_{i} \varepsilon_{i} \mid \sum_{i=1}^{p} x_{i}=0\right\} .
$$

The fundamental root system of $(\mathfrak{k}, \mathfrak{t})$ is

$$
\Pi(\mathfrak{k})=\left\{\alpha_{i}=\varepsilon_{i}-\varepsilon_{i+1} \mid i=1,2, \ldots, p-1\right\} .
$$

Set $\gamma_{i}^{*}=2 \gamma_{i} /\left(\gamma_{i}, \gamma_{i}\right)=\gamma_{i}, i=1,2, \ldots, p-1$.

The sets $\Gamma(S U(p)), Z(S U(p))$ and $D(S U(p))$ are given explicitly as follows:

$$
\begin{aligned}
\Gamma(S U(p))=\left\{2 \pi \sum_{i=1}^{p} x_{i} \varepsilon_{i} \mid\right. & \left.\sum_{i=1}^{p} x_{i}=0, x_{i} \in \boldsymbol{Z}, i=1,2, \ldots, p\right\}, \\
Z(S U(p))=\left\{\frac{1}{2 \pi} \sum_{i=1}^{p} m_{i} \varepsilon_{i} \mid\right. & \sum_{i=1}^{p} m_{i}=0, \\
& \left.m_{i}-m_{i+1} \in \boldsymbol{Z}, i=1,2, \ldots, p-1\right\},
\end{aligned}
$$




$$
\begin{aligned}
D(S U(p))=\left\{\frac{1}{2 \pi} \sum_{i=1}^{p} m_{i} \varepsilon_{i} \mid\right. & \sum_{i=1}^{p} m_{i}=0, \\
& \left.0 \leq m_{i}-m_{i+1} \in \boldsymbol{Z}, i=1,2, \ldots, p-1\right\} .
\end{aligned}
$$

Furthermore, $\Gamma\left(S U(p) / \boldsymbol{Z}_{p}\right), Z\left(S U(p) / \boldsymbol{Z}_{p}\right)$ and $D\left(S U(p) / \boldsymbol{Z}_{p}\right)$ are given explicitly as follows:

$$
\begin{aligned}
& \Gamma\left(S U(p) / \boldsymbol{Z}_{p}\right)=\left\{2 \pi \sum_{i=1}^{p} x_{i} \varepsilon_{i} \mid \sum_{i=1}^{p} x_{i}=0, x_{i}-x_{i+1} \in \boldsymbol{Z}, i=1,2, \ldots, p-1\right\}, \\
& Z\left(S U(p) / \boldsymbol{Z}_{p}\right)=\left\{\frac{1}{2 \pi} \sum_{i=1}^{p} m_{i} \varepsilon_{i} \mid \sum_{i=1}^{p} m_{i}=0, m_{i} \in \boldsymbol{Z}, i=1,2, \ldots, p\right\}, \\
& D\left(S U(p) / \boldsymbol{Z}_{p}\right)=\left\{\frac{1}{2 \pi} \sum_{i=1}^{p} m_{i} \varepsilon_{i} \mid \sum_{i=1}^{p} m_{i}=0, m_{i} \in \boldsymbol{Z}, i=1,2, \ldots, p,\right. \\
&\left.m_{i}-m_{i+1} \geq 0, i=1,2, \ldots, p-1\right\} .
\end{aligned}
$$

Here $\delta(G)$ is given by

$$
\delta(G)=\frac{1}{2 \pi} \frac{1}{2} \sum_{i=1}^{p}(p-2 i+1) \varepsilon_{i} .
$$

By the Freudenthal formula we determine all eigenvalues of the Casimir opetrator of $S U(p) / \boldsymbol{Z}_{p}$. For each $\Lambda \in D\left(S U(p) / \boldsymbol{Z}_{p}\right)$, the corresponding eigenvalue $-a_{\Lambda}$ is given by

$$
\begin{aligned}
-a_{\Lambda} & =4 \pi^{2}\left(\frac{1}{2 \pi} \sum_{i=1}^{p} m_{i} \varepsilon_{i}+\frac{1}{2 \pi} \sum_{i=1}^{p}(p-2 i+1) \varepsilon_{i}, \frac{1}{2 \pi} \sum_{i=1}^{p} m_{i} \varepsilon_{i}\right) \\
& =\sum_{i=1}^{p} m_{i}^{2}-2 \sum_{i=1}^{p} i m_{i} .
\end{aligned}
$$

For $\left(m_{1}, m_{2}, \ldots, m_{p}\right)=(1,0, \ldots, 0,-1)$, we have $-a_{\Lambda}=2-2(1-p)=2 p$.

By using Lemma 3.2, we see that $-a_{\Lambda}=2 p$ is the first eigenvalue of $S U(p) / Z_{p}$. By using Weyl's dimension formula (3.4), we can show that its multiplicity is equal to $p^{2}\left(p^{2}-\right.$ 1). Hence the first eigenvalue of $S U(p) / Z_{p}$ relative to the metric $(-1) B_{\mathfrak{k}}($,$) is equal to$ $2 p / 2 p=1$. Thus the first eigenvalue of $\left(S U(p) / \boldsymbol{Z}_{p} \times S U(p) / \boldsymbol{Z}_{p}\right) /\left(S U(p) / \boldsymbol{Z}_{p}\right)$ relative to the metric $(-1) B_{\mathfrak{g}}($,$) is equal to 1 \times 2=2$.

The case $G / K=S U(p) / S O(p) Z_{p}$ : Suppose that $\mathfrak{g}=\mathfrak{s u}(p)$ and $\mathfrak{k}=\mathfrak{s o}(p)$. Let $\left\{\varepsilon_{1}, \varepsilon_{2}, \ldots, \varepsilon_{p}\right\}$ be the standard orthonormal basis of $\boldsymbol{R}^{p}$.

Recall the Satake diagram of AI: 


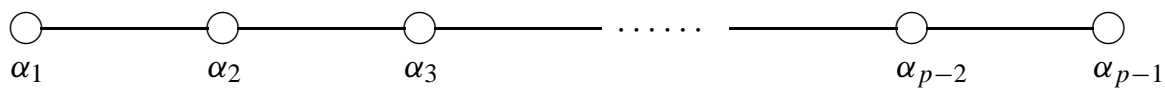

The fundamental root system of $(\mathfrak{g}, \mathfrak{t})$ can be found as follows:

$$
\Pi(\mathfrak{g})=\left\{\alpha_{i}=\varepsilon_{i}-\varepsilon_{i+1} \mid i=1,2, \ldots, p-1\right\} .
$$

In this case $\Pi_{0}(\mathfrak{g})=\emptyset, \mathfrak{b}=\{0\}$ and

$$
\Pi(\mathfrak{g}, \mathfrak{a})=\left\{\gamma_{i}=\bar{\alpha}_{i}=\alpha_{i} \mid i=1,2, \ldots, p-1\right\}=\Pi(\mathfrak{g}) .
$$

Then the subspace $\mathfrak{a}$ is given by

$$
\mathfrak{a}=\sum_{i=1}^{p-1} \boldsymbol{R} \cdot\left(\varepsilon_{i}-\varepsilon_{i+1}\right) .
$$

The Dynkin diagram of $\Pi(\mathfrak{g}, \mathfrak{a})$ is of type $A_{p-1}$ and given by

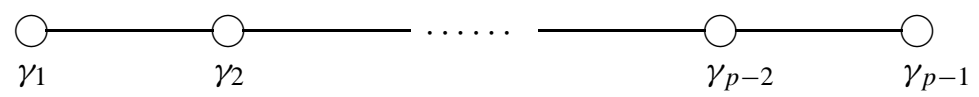

Set $\beta_{i}:=\gamma_{i}, i=1,2, \ldots, p-1$, and $\beta_{i}^{*}:=\gamma_{i}^{*}=2 \gamma_{i} /\left(\gamma_{i}, \gamma_{i}\right)=\gamma_{i}, i=1,2, \ldots, p-1$.

The sets $\Gamma(S U(p), S O(p)), Z(S U(p), S O(p))$ and $D(S U(p), S O(p))$ are described as follows:

$$
\begin{aligned}
& \Gamma(S U(p), S O(p))=\left\{\pi \sum_{i=1}^{p} x_{i} \varepsilon_{i} \mid \sum_{i=1}^{p} x_{i}=0, x_{i} \in \boldsymbol{Z}, i=1,2, \ldots, p\right\}, \\
& Z(S U(p), S O(p))=\left\{\frac{1}{\pi} \sum_{i=1}^{p} m_{i} \varepsilon_{i} \mid \sum_{i=1}^{p} m_{i}=0,\right. \\
& \left.m_{i}-m_{i+1} \in \boldsymbol{Z}, i=1,2, \ldots, p-1\right\}, \\
& D(S U(p), S O(p))=\left\{\frac{1}{\pi} \sum_{i=1}^{p} m_{i} \varepsilon_{i} \mid \begin{array}{l}
\sum_{i=1}^{p} m_{i}=0, \\
\left.0 \leq m_{i}-m_{i+1} \in \boldsymbol{Z}, i=1,2, \ldots, p-1\right\} .
\end{array}\right.
\end{aligned}
$$


Similarly, $\Gamma\left(S U(p), S O(p) Z_{p}\right), Z\left(S U(p), S O(p) Z_{p}\right)$ and $D\left(S U(p), S O(p) Z_{p}\right)$ are described as follows:

$$
\begin{aligned}
& \Gamma\left(S U(p), S O(p) \boldsymbol{Z}_{p}\right)=\left\{\pi \sum_{i=1}^{p} x_{i} \varepsilon_{i} \mid \sum_{i=1}^{p} x_{i}=0, x_{i}-x_{i+1} \in \boldsymbol{Z}, i=1,2, \ldots, p-1\right\}, \\
& Z\left(S U(p), S O(p) Z_{p}\right)=\left\{\frac{1}{\pi} \sum_{i=1}^{p} m_{i} \varepsilon_{i} \mid \sum_{i=1}^{p} m_{i}=0, m_{i} \in \boldsymbol{Z}, i=1,2, \ldots, p\right\}, \\
& D\left(S U(p), S O(p) Z_{p}\right)=\left\{\frac{1}{\pi} \sum_{i=1}^{p} m_{i} \varepsilon_{i} \mid \sum_{i=1}^{p} m_{i}=0, m_{i} \in \boldsymbol{Z}, i=1,2, \ldots, p,\right. \\
& \left.m_{i}-m_{i+1} \geq 0, i=1,2, \ldots, p-1\right\} \text {. }
\end{aligned}
$$

Here

$$
\delta(S U(p))=\frac{1}{2 \pi} \frac{1}{2} \sum_{i=1}^{p}(p-2 i+1) \varepsilon_{i} .
$$

For each $\Lambda \in D\left(S U(p), S O(p) Z_{p}\right)$, the corresponding eigenvalue $-a_{\Lambda}$ is

$$
\begin{aligned}
-a_{\Lambda} & =4 \pi^{2}\left(\frac{1}{\pi} \sum_{i=1}^{p} m_{i} \varepsilon_{i}+\frac{1}{2 \pi} \sum_{i=1}^{p}(p-2 i+1) \varepsilon_{i}, \frac{1}{\pi} \sum_{i=1}^{p} m_{i} \varepsilon_{i}\right) \\
& =4 \sum_{i=1}^{p}\left(m_{i}\right)^{2}-4 \sum_{i=1}^{p} i m_{i} .
\end{aligned}
$$

For $\left(m_{1}, m_{2}, \ldots, m_{p}\right)=(1,0, \ldots, 0,-1)$, we have $-a_{\Lambda}=4(p+1)$. This is the first eigenvalue of $S U(p) / S O(p) Z_{p}$ by Lemma 3.2. The first eigenvalue of $S U(p) / S O(p) \boldsymbol{Z}_{p}$ relative to the metric $(-1) B_{\mathfrak{g}}($,$) is equal to 2(p+1) / p$. The first eigenvalue of $S U(p) / S O(p) Z_{p}$ relative to the metric $(-2) B_{\mathfrak{g}}(,) / p^{2}$ is equal to $p(p+1) / 4$. By using Weyl's dimension formula (3.4), we can show that its multiplicity is equal to $p^{2}(p-1)(p+3) / 4$.

The case $G / K=S U(2 p) / S p(p) Z_{2 p}$ : Suppose that $\mathfrak{g}=\mathfrak{s u}(2 p)$ and $\mathfrak{k}=\mathfrak{s p}(p)$. Let $\left\{\varepsilon_{1}, \varepsilon_{2}, \ldots, \varepsilon_{2 p}\right\}$ be the standard orthonormal basis of $\boldsymbol{R}^{2 p}$. The subspace $\mathfrak{t}$ can be identified with

$$
\mathfrak{t}=\left\{\sum_{i=1}^{2 p} \xi^{i} \varepsilon_{i} \mid \sum_{i=1}^{2 p} \xi^{i}=0\right\} .
$$

Recall the Satake diagram of AII: 


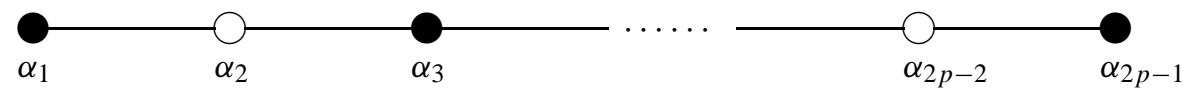

The fundamental root system of $(\mathfrak{g}, \mathfrak{k})$ is given by

$$
\Pi(\mathfrak{g})=\left\{\alpha_{i}=\varepsilon_{i}-\varepsilon_{i+1} \mid i=1,2, \ldots, 2 p-1\right\} .
$$

In this case

$$
\begin{gathered}
\Pi_{0}(\mathfrak{g})=\left\{\alpha_{2 i-1} \mid i=1,2, \ldots, p\right\} \\
\mathfrak{b}=\sum_{i=1}^{p} \boldsymbol{R} \cdot \alpha_{2 i-1}=\sum_{i=1}^{p} \boldsymbol{R} \cdot\left(\varepsilon_{2 i-1}-\varepsilon_{2 i}\right)
\end{gathered}
$$

and

$$
\Pi(\mathfrak{g}, \mathfrak{a})=\left\{\gamma_{i}=\bar{\alpha}_{2 i} \mid i=1,2, \ldots, p-1\right\}
$$

The Dynkin diagram of $\Pi(\mathfrak{g}, \mathfrak{a})$ is of type $A_{p-1}$ :

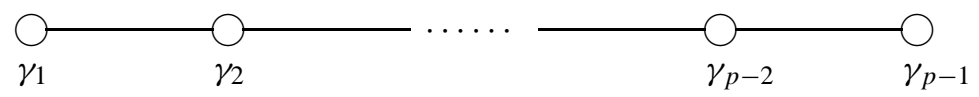

Define $p$ vectors $f_{i}$ of $\boldsymbol{R}^{2 p}$ by

$$
f_{i}:=\frac{1}{\sqrt{2}}\left(\varepsilon_{2 i-1}+\varepsilon_{2 i}\right), \quad i=1,2, \ldots, p-1, p .
$$

Then, for $i=1,2, \ldots, p-1$ we have

$$
\alpha_{2 i}=\varepsilon_{2 i}-\varepsilon_{2 i+1}, \quad \gamma_{i}=\frac{1}{\sqrt{2}}\left(f_{i}-f_{i+1}\right) .
$$

The subspace $\mathfrak{a}$ can be identified with

$$
\mathfrak{a}=\sum_{i=1}^{p-1} \boldsymbol{R} \cdot\left(f_{i}-f_{i+1}\right) .
$$


Set $\beta_{i}:=\gamma_{i}$, and $\beta_{i}^{*}:=\gamma_{i}^{*}=2 \gamma_{i} /\left(\gamma_{i}, \gamma_{i}\right)=2 \gamma_{i}, i=1,2, \ldots, p-1$. Then $\Gamma(S U(2 p), S p(p)), Z(S U(2 p), S p(p))$ and $D(S U(2 p), S p(p))$ are described as follows:

$$
\begin{aligned}
& \Gamma(S U(2 p), S p(p))=\left\{\sqrt{2} \pi \sum_{i=1}^{p} x_{i} f_{i} \mid \sum_{i=1}^{p} x_{i}=0, x_{i} \in \boldsymbol{Z}, i=1,2, \ldots, p\right\}, \\
& Z(S U(2 p), S p(p))=\left\{\frac{1}{\sqrt{2} \pi} \sum_{i=1}^{p} m_{i} f_{i} \mid \sum_{i=1}^{p} m_{i}=0,\right. \\
& \left.m_{i}-m_{i+1} \in \boldsymbol{Z}, i=1,2, \ldots, p-1\right\}, \\
& D(S U(2 p), S p(p))=\left\{\frac{1}{\sqrt{2} \pi} \sum_{i=1}^{p} m_{i} f_{i} \mid \sum_{i=1}^{p} m_{i}=0,\right. \\
& \left.0 \leq m_{i}-m_{i+1} \in \boldsymbol{Z}, i=1,2, \ldots, p-1\right\} .
\end{aligned}
$$

Moreover, $\Gamma\left(S U(2 p), S p(p) \boldsymbol{Z}_{2 p}\right), Z\left(S U(2 p), S p(p) \boldsymbol{Z}_{2 p}\right)$ and $D\left(S U(2 p), S p(p) \boldsymbol{Z}_{2 p}\right)$ are described as follows:

$$
\begin{aligned}
& \Gamma\left(S U(2 p), S p(p) \boldsymbol{Z}_{2 p}\right)=\left\{\sqrt{2} \pi \sum_{i=1}^{p} x_{i} f_{i} \mid \sum_{i=1}^{p} x_{i}=0,\right. \\
& \left.x_{i}-x_{i+1} \in \boldsymbol{Z}, i=1,2, \ldots, p-1\right\}, \\
& Z\left(S U(2 p), S p(p) \boldsymbol{Z}_{2 p}\right)=\left\{\frac{1}{\sqrt{2} \pi} \sum_{i=1}^{p} m_{i} f_{i} \mid \sum_{i=1}^{p} m_{i}=0, m_{i} \in \boldsymbol{Z}, i=1,2, \ldots, p\right\}, \\
& D\left(S U(2 p), S p(p) Z_{2 p}\right)=\left\{\frac{1}{\sqrt{2} \pi} \sum_{i=1}^{p} m_{i} f_{i} \mid \sum_{i=1}^{p} m_{i}=0, m_{i} \in \boldsymbol{Z}, i=1,2, \ldots, p,\right. \\
& \left.m_{i}-m_{i+1} \geq 0, i=1,2, \ldots, p-1\right\} .
\end{aligned}
$$

Here

$$
\begin{aligned}
\delta(S U(2 p)) & =\frac{1}{2 \pi} \frac{1}{2} \sum_{i=1}^{2 p}(2 p-2 i+1) \varepsilon_{i} \\
& =\frac{1}{2 \pi} \frac{1}{2} \sum_{i=1}^{p}\left((2 p-4 i+3) \varepsilon_{2 i-1}+(2 p-4 i+1) \varepsilon_{2 i}\right) .
\end{aligned}
$$


For each $\Lambda \in D\left(S U(2 p), S p(p) Z_{2 p}\right)$, the corresponding eigenvalue $-a_{\Lambda}$ is

$$
\begin{aligned}
-a_{\Lambda} & =4 \pi^{2}\left(\frac{1}{\sqrt{2} \pi} \sum_{i=1}^{p} m_{i} f_{i}+\frac{1}{2 \pi} \sum_{i=1}^{2 p}(2 p-2 i+1) \varepsilon_{i}, \frac{1}{\sqrt{2} \pi} \sum_{i=1}^{p} m_{i} f_{i}\right) \\
& =2 \sum_{i=1}^{p}\left(m_{i}\right)^{2}-8 \sum_{i=1}^{p} i m_{i} .
\end{aligned}
$$

All eigenvalues of the Laplacian on functions for $S U(2 p) / S p(p) Z_{2 p}$ relative to the metric $(-1) B_{\mathfrak{g}}($,$) are given by the formula$

$$
-\frac{1}{4 p} a_{\Lambda}=\frac{1}{2 p}\left(\sum_{i=1}^{p}\left(m_{i}\right)^{2}-4 \sum_{i=1}^{p} i m_{i}\right)=\frac{1}{2 p} \sum_{i=1}^{p} m_{i}\left(m_{i}-4 i\right) .
$$

For $\left(m_{1}, m_{2}, \ldots, m_{p}\right)=(1,0, \ldots, 0,-1)$,

$$
-\frac{1}{4 p} a_{\Lambda}=\frac{1}{2 p} \sum_{i=1}^{p} m_{i}\left(m_{i}-4 i\right)=\frac{2 p-1}{p} .
$$

By using Lemma 3.2, we see that $-a_{\Lambda} / 4 p=(2 p-1) / p$ is the first eigenvalue of $S U(2 p) /$ $S p(p) Z_{2 p}$. By Weyl's dimension formula (3.4), we can show that its multiplicity is equal to $p^{2}(2 p+1)(2 p-3)$.

The case $G / K=E_{6} / F_{4} Z_{3}$ : Suppose that $\mathfrak{g}=\mathfrak{e}_{6}$ and $\mathfrak{k}=\mathfrak{f}_{4}$. Let $\left\{\varepsilon_{1}, \varepsilon_{2}, \ldots, \varepsilon_{8}\right\}$ be the standard orthonormal basis of $\boldsymbol{R}^{8}$. Let

$$
\mathfrak{t}=\sum_{i=1}^{5} \boldsymbol{R} \cdot \varepsilon_{i}+\boldsymbol{R} \cdot\left(\varepsilon_{6}+\varepsilon_{7}-\varepsilon_{8}\right) \subset \boldsymbol{R}^{8} .
$$

The fundamental root system of $(\mathfrak{g}, \mathfrak{t})$ is given as

$$
\Pi(\mathfrak{g})=\left\{\alpha_{1}, \alpha_{2}, \ldots, \alpha_{6}\right\},
$$

where

$$
\begin{aligned}
\alpha_{1} & =\frac{1}{2}\left(\varepsilon_{1}+\varepsilon_{8}\right)-\frac{1}{2}\left(\varepsilon_{2}+\varepsilon_{3}+\varepsilon_{4}+\varepsilon_{5}+\varepsilon_{6}+\varepsilon_{7}\right), \\
\alpha_{2} & =\varepsilon_{1}+\varepsilon_{2}, \\
\alpha_{3} & =\varepsilon_{2}-\varepsilon_{1}, \\
\alpha_{4} & =\varepsilon_{3}-\varepsilon_{2}, \\
\alpha_{5} & =\varepsilon_{4}-\varepsilon_{3}, \\
\alpha_{6} & =\varepsilon_{5}-\varepsilon_{4} .
\end{aligned}
$$

The Satake diagram of EIV is given by 


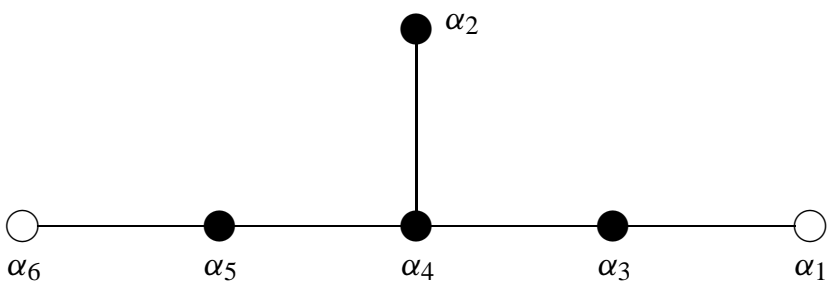

Moreover,

$$
\Pi_{0}(\mathfrak{g})=\left\{\alpha_{2}, \alpha_{3}, \alpha_{4}, \alpha_{5}\right\}
$$

and

$$
\begin{aligned}
\mathfrak{b} & =\boldsymbol{R} \cdot \alpha_{2}+\boldsymbol{R} \cdot \alpha_{3}+\boldsymbol{R} \cdot \alpha_{4}+\boldsymbol{R} \cdot \alpha_{5} \\
& =\boldsymbol{R} \cdot \varepsilon_{1}+\boldsymbol{R} \cdot \varepsilon_{2}+\boldsymbol{R} \cdot \varepsilon_{3}+\boldsymbol{R} \cdot \varepsilon_{4}, \\
\mathfrak{a} & =\boldsymbol{R} \cdot \varepsilon_{5}+\boldsymbol{R} \cdot\left(\varepsilon_{6}+\varepsilon_{7}-\varepsilon_{8}\right) .
\end{aligned}
$$

The fundamental root system of $(\mathfrak{g}, \mathfrak{a})$ is given as

$$
\Pi(\mathfrak{g}, \mathfrak{a})=\left\{\bar{\alpha}_{1}, \bar{\alpha}_{6}\right\}
$$

Set

$$
\gamma_{1}:=\bar{\alpha}_{1}=-\frac{1}{2}\left(\varepsilon_{5}+\varepsilon_{6}+\varepsilon_{7}-\varepsilon_{8}\right), \quad \gamma_{2}:=\bar{\alpha}_{6}=\varepsilon_{5} .
$$

The Dynkin diagram of $\Pi(\mathfrak{g}, \mathfrak{a})$ is of type $A_{2}$ and is given by

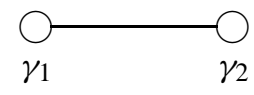

Define

$$
\gamma_{1}^{*}:=\frac{2}{\left(\gamma_{1}, \gamma_{1}\right)} \gamma_{1}=2 \gamma_{1}, \quad \gamma_{2}^{*}:=\frac{2}{\left(\gamma_{2}, \gamma_{2}\right)} \gamma_{2}=2 \gamma_{2} .
$$

Let $\left\{f_{1}, f_{2}\right\}$ be an orthonormal basis of $\mathfrak{a}$ defined by

$$
f_{1}=\varepsilon_{5}, \quad f_{2}=-\frac{1}{\sqrt{3}}\left(\varepsilon_{6}+\varepsilon_{7}-\varepsilon_{8}\right) .
$$

Then we have

$$
\gamma_{1}=-\frac{1}{2} f_{1}+\frac{\sqrt{3}}{2} f_{2}, \quad \gamma_{2}=f_{1}
$$


Moreover, we have

$$
\begin{aligned}
\Gamma\left(E_{6}, F_{4}\right) & =\left\{\pi\left(k_{1} f_{1}+\sqrt{3} k_{2} f_{2}\right) \mid k_{1}, k_{2} \in 2 \boldsymbol{Z} \text { or } k_{1}, k_{2} \in 2 \boldsymbol{Z}+1\right\}, \\
Z\left(E_{6}, F_{4}\right) & =\left\{x_{1} f_{1}+x_{2} f_{2} \mid x_{1} \in \frac{1}{2 \pi} Z, x_{2} \in \frac{1}{2 \sqrt{3} \pi} \boldsymbol{Z}, x_{1}+\sqrt{3} x_{2} \in \frac{1}{\pi} \boldsymbol{Z}\right\} \\
& =\left\{\frac{1}{2 \pi}\left(k_{1} f_{1}+\frac{1}{\sqrt{3}} k_{2} f_{2}\right) \mid k_{1}, k_{2} \in \boldsymbol{Z}, k_{1}+k_{2} \in 2 \boldsymbol{Z}\right\}, \\
D\left(E_{6}, F_{4}\right) & =\left\{\frac{1}{2 \pi}\left(k_{1} f_{1}+\frac{1}{\sqrt{3}} k_{2} f_{2}\right) \mid k_{1}, k_{2} \in \boldsymbol{Z}, k_{1}+k_{2} \in 2 \boldsymbol{Z}, k_{2} \geq k_{1} \geq 0\right\} .
\end{aligned}
$$

Similarly $\Gamma\left(E_{6}, F_{4} \boldsymbol{Z}_{3}\right), Z\left(E_{6}, F_{4} \boldsymbol{Z}_{3}\right)$ and $D\left(E_{6}, F_{4} \boldsymbol{Z}_{3}\right)$ are described as follows:

$$
\begin{aligned}
& \Gamma\left(E_{6}, F_{4} \boldsymbol{Z}_{3}\right)=\left\{\pi\left(k_{1} f_{1}+\frac{k_{2}}{\sqrt{3}} f_{2}\right) \mid k_{1}, k_{2} \in 2 \boldsymbol{Z} \text { or } k_{1}, k_{2} \in 2 \boldsymbol{Z}+1\right\} \\
& Z\left(E_{6}, F_{4} \boldsymbol{Z}_{3}\right)=\left\{x_{1} f_{1}+x_{2} f_{2} \mid x_{1} \in \frac{1}{2 \pi} \boldsymbol{Z}, x_{2} \in \frac{\sqrt{3}}{2 \pi} \boldsymbol{Z}, x_{1}+\frac{1}{\sqrt{3}} x_{2} \in \frac{1}{\pi} \boldsymbol{Z}\right\} \\
&=\left\{\frac{1}{2 \pi}\left(k_{1} f_{1}+\frac{1}{\sqrt{3}} k_{2} f_{2}\right) \mid k_{1} \in \boldsymbol{Z}, k_{2} \in 3 \boldsymbol{Z}, k_{1}+k_{2} \in 2 \boldsymbol{Z}\right\}, \\
& D\left(E_{6}, F_{4} \boldsymbol{Z}_{3}\right)=\left\{\frac{1}{2 \pi}\left(k_{1} f_{1}+\frac{1}{\sqrt{3}} k_{2} f_{2}\right) \mid k_{1} \in \boldsymbol{Z}, k_{2} \in 3 \boldsymbol{Z}, k_{1}+k_{2} \in 2 \boldsymbol{Z},\right. \\
&\left.k_{2} \geq k_{1} \geq 0\right\} .
\end{aligned}
$$

Here

$$
\begin{aligned}
\delta & =\frac{1}{2 \pi}\left(\varepsilon_{2}+2 \varepsilon_{3}+3 \varepsilon_{4}+4 \varepsilon_{5}+4\left(\varepsilon_{8}-\varepsilon_{7}-\varepsilon_{6}\right)\right) \\
& =\frac{1}{2 \pi}\left(\varepsilon_{2}+2 \varepsilon_{3}+3 \varepsilon_{4}+4 f_{1}+4 \sqrt{3} f_{2}\right) .
\end{aligned}
$$

Note that $\varepsilon_{2}+2 \varepsilon_{3}+3 \varepsilon_{4} \in \mathfrak{b}$ and $4 f_{1}+4 \sqrt{3} f_{2} \in \mathfrak{a}$.

For each $\Lambda=\Lambda\left(k_{1}, k_{2}\right) \in D\left(E_{6}, F_{4}\right)$, the corresponding eigenvalue $-a_{\Lambda}$ of $E_{6} / F_{4}$ is found by the following formula:

$$
-a_{\Lambda}=k_{1}\left(k_{1}+8\right)+k_{2}\left(\frac{k_{2}}{3}+8\right) .
$$

Here we examine eigenvalues corresponding to small values of pairs $\left(k_{1}, k_{2}\right)$. For $\left(k_{1}, k_{2}\right)=(1,1)$ or $\left(k_{1}, k_{2}\right)=(0,2)$, we have

$$
-a_{\Lambda}=\frac{52}{3} \quad \text { and } \quad-\frac{a_{\Lambda}}{24}=\frac{13}{18} \text {. }
$$

For $\left(k_{1}, k_{2}\right)=(2,2)$, we have

$$
-a_{\Lambda}=\frac{112}{3} \text { and } \quad-\frac{a_{\Lambda}}{24}=\frac{14}{9} .
$$


For $\left(k_{1}, k_{2}\right)=(1,3)$, we have

$$
-a_{\Lambda}=36 \text { and } \quad-\frac{a_{\Lambda}}{24}=\frac{3}{2} .
$$

For $\left(k_{1}, k_{2}\right)=(3,3)$, we have

$$
-a_{\Lambda}=60 \text { and } \quad-\frac{a_{\Lambda}}{24}=\frac{5}{2} .
$$

Otherwise, we obtain

$$
-\frac{a_{\Lambda}}{24}>\frac{3}{2}
$$

The first eigenvalue of $E_{6} / F_{4}$ is equal to $13 / 18$. The second eigenvalue of $E_{6} / F_{4}$ and the first eigenvalue of $E_{6} / F_{4} Z_{3}$ are equal to $3 / 2$. By Weyl's dimension formula (3.4), we can show that the multiplicity of the first eigenvalue of $E_{6} / F_{4} Z_{3}$ is equal to 650 .

LEMMA 3.2. Let $\left(m_{1}, \ldots, m_{p}\right)$ be a p-tuple of integers satisfying

$$
\sum_{i=1}^{p} m_{i}=0 \text { and } m_{i}-m_{i+1} \geq 0, i=1,2, \ldots, p .
$$

Suppose that $\left(m_{1}, \ldots, m_{p}\right) \neq 0$ and $t$ is a positive real number. Then the following inequality holds:

$$
\sum_{i=1}^{p}\left(m_{i}\right)^{2}-t \sum_{i=1}^{p} i m_{i} \geq 2+t(p-1)\left(m_{1}-m_{p}\right) / 2 .
$$

The equality holds if and only if $\left(m_{1}, m_{2}, \ldots, m_{p-1}, m_{p}\right)=(1,0, \ldots, 0,-1)$.

Proof. Put $\boldsymbol{Z} \ni k_{i}:=m_{i}-m_{i+1} \geq 0, i=1,2, \ldots, p-1$. Then we have $m_{p}=$ $-\sum_{j=1}^{p-1} j k_{j} / p$. By using the identity

$$
\begin{aligned}
\sum_{i=1}^{p} i m_{i} & =\sum_{i=1}^{p} i\left(m_{p}+k_{p-1}+k_{p-2}+\cdots+k_{i}\right) \\
& =m_{p} \frac{p(p+1)}{2}+\sum_{j=1}^{p-1} \frac{j(j+1)}{2} k_{j} \\
& =-\frac{p+1}{2} \sum_{j=1}^{p-1} j k_{j}+\sum_{j=1}^{p-1} \frac{j(j+1)}{2} k_{j} \\
& =-\frac{1}{2} \sum_{j=1}^{p-1} j(p-j) k_{j},
\end{aligned}
$$


we obtain

$$
\begin{aligned}
\sum_{i=1}^{p} m_{i}^{2}-t \sum_{i=1}^{p} i m_{i} & =\sum_{i=1}^{p} m_{i}^{2}+\frac{t}{2} \sum_{j=1}^{p-1} j(p-j) k_{j} \\
& \geq 2+\frac{t}{2} \sum_{j=1}^{p-1}(p-1) k_{j}=2+t(p-1)\left(m_{1}-m_{p}\right) / 2
\end{aligned}
$$

Here we see that

$$
\sum_{i=1}^{p} m_{i}^{2}+\frac{t}{2} \sum_{j=1}^{p-1} j(p-j) k_{j}=2+t(p-1)\left(m_{1}-m_{p}\right) / 2
$$

if and only if $\left(k_{1}, k_{2}, \ldots, k_{p-2}, k_{p-1}\right)=(1,0, \ldots, 0,1)(p \geq 3)$ or $k_{1}=2(p=2)$, and equivalently $\left(m_{1}, m_{2}, \ldots, m_{p-2}, m_{p-1}, m_{p}\right)=(1,0, \ldots, 0,0,-1)$.

4. Hamiltonian stability of minimal Lagrangian submanifolds in $C P^{n}$ with parallel second fundamental form. First, combining Proposition 2.1 and Lemma 3.1, we obtain the following lemma.

LEMMA 4.1. Suppose that $L$ is an $n$-dimensional compact totally real submanifold embedded in $C P^{n}$ constructed in Section 2 with $s=1$. Then the Einstein constant, the first eigenvalue $\lambda_{1}$ of the Laplacian acting on $C^{\infty}(L)$ with respect to the induced metric and its multiplicity $d_{1}$ are given in the following table:

\begin{tabular}{|c|c|c|c|c|c|}
\hline$L$ & $\widetilde{L}$ & $\kappa_{L}$ & $\lambda_{1}$ & $\tilde{\lambda}_{1}$ & $d_{1}$ \\
\hline $\boldsymbol{R} P^{n}$ & $S^{n}$ & $\frac{(n-1) c}{4}$ & $\frac{(n+1) c}{2}$ & $\frac{n c}{4}$ & $\frac{n(n+3)}{2}$ \\
\hline$\frac{S U(p)}{\boldsymbol{Z}_{p}}$ & $S U(p)$ & $\frac{p^{2} c}{8}$ & $\frac{p^{2} c}{2}$ & $\frac{\left(p^{2}-1\right) c}{2}$ & $\left(p^{2}-1\right)^{2}$ \\
\hline$\frac{S U(p)}{S O(p) \boldsymbol{Z}_{p}}$ & $\frac{S U(p)}{S O(p)}$ & $\frac{p^{2} c}{16}$ & $\frac{p(p+1) c}{4}$ & $\frac{(p+2)(p-1) c}{8}$ & $\frac{p^{2}(p-1)(p+3)}{4}$ \\
\hline$\frac{S U(2 p)}{S p(p) \boldsymbol{Z}_{2 p}}$ & $\frac{S U(2 p)}{S p(p)}$ & $\frac{p^{2} c}{4}$ & $\frac{p(2 p-1) c}{2}$ & $\frac{(p-1)(2 p+1) c}{4}$ & $p^{2}(2 p+1)(2 p-3)$ \\
\hline$\frac{E_{6}}{F_{4} \boldsymbol{Z}_{3}}$ & $\frac{E_{6}}{F_{4}}$ & $\frac{9 c}{2}$ & $\frac{27 c}{2}$ & $\frac{13 c}{2}$ & 650 \\
\hline
\end{tabular}

Here $\kappa_{L}$ is the Einstein constant of $L$ and $p \geq 2$ is an integer.

Comparing tables of Lemma 3.1 and 4.1, we see that $L$ satisfies the Hamiltonian stability condition of Theorem 1.2, that is, the first eigenvalue $\lambda_{1}$ of the Laplacian of $L$ is equal to $c(n+1) / 2$ in each case. We also note that this condition is not satisfied for $\widetilde{L}$. 
In Lemma 4.1, the multiplicies $d_{1}$ of $\lambda_{1}$ were computed by the Weyl's dimension formula (3.4). In these cases we have

$$
n(L)=d_{1}
$$

In each case $L=G / K$, we can observe the fact that

$$
\begin{aligned}
d_{1} & =\operatorname{dim}(\mathfrak{s u}(n+1))-\operatorname{dim} G \\
& =\operatorname{dim} \mathcal{K}-\operatorname{dim} I_{0}(L) .
\end{aligned}
$$

By (1.2), we obtain

$$
n(L)=n_{\mathcal{K}}(L) .
$$

Therefore we obtain the following theorem.

THEOREM 4.1. Let $L$ be an $n$-dimensional compact totally real minimal submanifold embedded in $\boldsymbol{C P}^{n}$ with parallel second fundamental form in the following list:

(1) $S U(p) / Z_{p}, n=p^{2}-1$.

(2) $S U(p) / S O(p) Z_{p}, n=(p-1)(p+2) / 2$.

(3) $S U(2 p) / S p(p) Z_{2 p}, n=(p-1)(2 p+1)$.

(4) $E_{6} / F_{4} Z_{3}, n=26$.

Then $L$ is a Hamiltonian stable minimal Lagrangian submanifold in $\boldsymbol{C P}^{n}$. Moreover the null space of $L$ is exactly the span of the normal projections of Killing vector fields on $C P^{n}$.

In our cases we see that all the eigenfunctions of the Laplacian on $L$ with eigenvalue $c(n+1) / 2$ are obtained as the restriction of the first eigenfunctions of the Laplacian on $C P^{n}$ to $L$.

The submanifolds from (1) through (4) of Theorem 4.1 and the real projective space $\boldsymbol{R} P^{n}$ exhaust all compact irreducible totally real minimal submanifolds embedded in the complex projective space $\boldsymbol{C} P^{n}$ with parallel second fundamental form. Hence we state the following

THEOREM 4.2. All n-dimensional compact totally real minimal submanifolds embedded in $\boldsymbol{C} P^{n}$ with parallel second fundamental form and positive Ricci curvature are Hamiltonian stable as minimal Lagrangian submanifolds.

Based on our results we shall now remark on some related open problems.

PROBLEM 4.1. Is it true that all compact $n$-dimensional totally real submanifolds embedded in $\boldsymbol{C} P^{n}$ with parallel second fundamental form are Hamiltonian stable as H-minimal Lagrangian submanifolds?

Problem 4.2. Is it true that compact Hamiltonian stable H-minimal Lagrangian submanifolds in $\boldsymbol{C} P^{n}$ have parallel second fundamental form?

Problem 4.3. Is such a compact Hamiltonian stable H-minimal Lagrangian submanifold $L$ in $C P^{n}$ globally Hamiltonian stable or not, that is, volume minimizing with respect to every Hamiltonian deformation of $L$ ? 
Few examples of globally Hamiltonian stable (H-)minimal Lagrangian submanifolds seems to be known in specific Kähler manifolds such as complex projective spaces, compact Hermitian symmetric spaces and so on. It is known that the real projective subspaces $\boldsymbol{R} P^{n} \subset \boldsymbol{C} P^{n}$ are globally Hamiltonian stable minimal Lagrangian submanifolds in $\boldsymbol{C} P^{n}$ (cf. [1] and [18]).

5. Hamiltonian stability of symmetric $R$-spaces canonically embedded in Hermitian symmetric spaces. In this section we discuss Hamiltonian stability of totally geodesic Lagrangian submanifolds in compact Hermitian symmetric spaces of rank greater than 1. Let $M$ be a compact Hermitian symmetric space and $L$ be a symmetric $R$-space canonically embedded in $M$. Such pairs represent a nice class of minimal Lagrangian submanifolds embedded in Einstein-Kähler manifolds.

A real form of a compact Hermitian symmetric space $M$ is defined as the fixed point subset by an involutive anti-holomorphic isometry. It is a totally real and totally geodesic submanifold of $M$ with dimension equal to the half of $\operatorname{dim} M$, and hence it is a totally geodesic Lagrangian submanifold of $M$. In [25], Takeuchi proved that every compact totally geodesic Lagrangian submanifold of a compact Hermitian symmetric space $M$ is obtained as a real form by an involutive anti-holomorphic isometry of $M$, and it is nothing but a symmetric $R$-space canonically embedded in a compact Hermitian symmetric space. Moreover in [25], he showed that a symmetric $R$-space $L$ canonically embedded in a compact Hermitian symmetric space is stable if and only if $L$ is simply connected. The theory of symmetric $R$-spaces is wellinvestigated and we refer [25] for a complete list of symmetric $R$-spaces. In [25], he also proved the following.

THEOREM 5.1 ([25]). Let L be a symmetric $R$-space canonically embedded in a compact irreducible Hermitian symmetric space $M=G / K$ equipped with a metric $g$ induced from the (-1)-times Killing-Cartan form of $\mathfrak{g}=\operatorname{Lie}(G)$. If $g$ is an Einstein metric, then the first eigenvalue of the Laplacian of $L$ acting on $C^{\infty}(L)$ is equal to $1 / 2$.

Moreover, we already know that a compact irreducible Hermitian symmetric space $M$ with a metric $g$ induced from the (-1)-times Killing-Cartan form is an Einstein manifold with Einstein constant $1 / 2$. Hence Theorem 5.1 implies that, if an irreducible symmetric $R$-space embedded in a compact Hermitian symmetric space as a real form is an Einstein manifold, then it is a Hamiltonian stable minimal Lagrangian submanifold (see [18]). Analyzing root space of symmetric $R$-spaces, Takeuchi classified all irreducible symmetric $R$-spaces into five classes: The Hermitian and four other types (cf. [25]). The irreducible symmetric $R$-spaces of Hermitian type are just irreducible Hermitian symmetric spaces of compact type, which are Einstein. The Hermitian symmetric spaces of compact type canonically embedded in a Hermitian symmetric space $M$ as a real form are always stable and hence Hamiltonian stable. By calculating the first eigenvalues of symmetric $R$-spaces of other types canonically embedded in a compact irreducible Hermitian symmetric space, we can determine their Hamiltonian 
stability. Here we give a complete list of the Hamiltonian stability for all irreducible symmetric $R$-spaces of non-Hermitian type canonically embedded in compact irreducible Hermitian symmetric spaces.

\begin{tabular}{|c|c|c|c|c|c|}
\hline$M$ & $L$ & Einstein & $\mu_{1}$ & H-stable & stable \\
\hline$G_{p, q}(\boldsymbol{C}), q \geq p \geq 1$ & $G_{p, q}(\boldsymbol{R})$ & Yes & $1 / 2$ & Yes & No \\
\hline$G_{2 p, 2 q}(\boldsymbol{C}), q \geq p \geq 1$ & $G_{p, q}(\boldsymbol{H})$ & Yes & $1 / 2$ & Yes & Yes \\
\hline$G_{m, m}(\boldsymbol{C}), m \geq 2$ & $U(m)$ & No & $1 / 2$ & Yes & No \\
\hline$\frac{S O(2 m)}{U(m)}$ & $S O(m), m \geq 5$ & Yes & $1 / 2$ & Yes & No \\
\hline$\frac{S O(4 m)}{U(2 m)}, m \geq 3$ & $\frac{U(2 m)}{S p(m)}$ & No & $\frac{m}{4 m-2}$ & No & No \\
\hline$\frac{S p(2 m)}{U(2 m)}$ & $S p(m), m \geq 2$ & Yes & $1 / 2$ & Yes & Yes \\
\hline$\frac{S p(m)}{U(m)}, m \geq 3$ & $\frac{U(m)}{O(m)}$ & No & $1 / 2$ & Yes & No \\
\hline$Q_{p+q-2}(\boldsymbol{C}), q-p \geq 3$ & $Q_{p, q}(\boldsymbol{R}), p \geq 2$ & No & $\frac{p}{p+q-2}$ & No & No \\
\hline$Q_{2 p-2}(\boldsymbol{C})$ & $Q_{p, p}(\boldsymbol{R}), p \geq 2$ & Yes & $1 / 2$ & Yes & No \\
\hline$Q_{2 p+k-2}(\boldsymbol{C}), k=1,2$ & $Q_{p, p+k}(\boldsymbol{R}), p \geq 2$ & No & $1 / 2$ & Yes & No \\
\hline$Q_{q}(\boldsymbol{C}), q<5$ & $Q_{2, q}(\boldsymbol{R})$ & No & $1 / 2$ & Yes & No \\
\hline$Q_{q-1}(\boldsymbol{C}), q<4$ & $Q_{1, q}(\boldsymbol{R})$ & Yes & $1 / 2$ & Yes & No \\
\hline$E_{6} / T \cdot S p i n(10)$ & $P_{2}(\boldsymbol{K})$ & Yes & $1 / 2$ & Yes & Yes \\
\hline$E_{6} / T \cdot \operatorname{Spin}(10)$ & $G_{2,2}(\boldsymbol{H}) / \boldsymbol{Z}_{2}$ & Yes & $1 / 2$ & Yes & No \\
\hline$E_{7} / T \cdot E_{6}$ & $S U(8) / S p(4) Z_{2}$ & Yes & $1 / 2$ & Yes & No \\
\hline$E_{7} / T \cdot E_{6}$ & $T \cdot E_{6} / F_{4}$ & No & $1 / 6$ & No & No \\
\hline
\end{tabular}


Here we use the following notation:

$G_{p, q}(\boldsymbol{F})$ : Grassmann manifold of all $p$-dimensional subspaces of $\boldsymbol{F}^{p+q}$ for $\boldsymbol{F}=\boldsymbol{R}, \boldsymbol{C}$ and a real quaternion algebra $\boldsymbol{H}$.

$\boldsymbol{P}_{2}(K)$ : Cayley projective plane,

$Q_{n}(\boldsymbol{C})$ : complex quadric of dimension $n$,

$Q_{p, q}(\boldsymbol{R})=\left\{[x] \in \boldsymbol{R} P^{p+q-1} \mid x_{1}^{2}+\cdots+x_{p}^{2}-x_{p+1}^{2}-\cdots-x_{p+q}^{2}=0\right\}$ : real quadric.

Note that the third column indicates whether $L$ is Einstein or not.

\section{REFERENCES}

[1] A. Amarzaya And Y. OhnitA, On Hamiltonian stability of certain H-minimal Lagrangian submanifolds in Hermitian symmetric spaces, RIMS Kokyuroku 1236, Geometry of Submanifolds and Related Topics, 2001, RIMS, Kyoto University, Kyoto Japan, 31-48.

[2] A. AmarzayA And Y. OHnitA, Hamiltonian stability of certain symmetric $R$-spaces embedded in complex Euclidean spaces, preprint, Tokyo Metropolitan University, 2002.

[ 3 ] N. Bourbaki, Éléments de mathématique. Fasc. XXXIV. Groupes et algebres de Lie, Actualites Scientifiques et Industrielles, No. 1337, Hermann, Paris, 1968.

[ 4 ] S. Chang, On Hamiltonian stable minimal Lagrangian surfaces in $\boldsymbol{C} P^{2}$, J. Geom. Anal. 10 (2000), $243-255$.

[ 5 ] B. Y. Chen, Geometry of Submanifolds and its Applications, Science University of Tokyo, 1981.

[6] B. Y. Chen AND K. OGiue, On totally real submanifolds, Trans. Amer. Math. Soc. 193 (1974), $257-266$.

[ 7 ] P. DAZORD, Sur la géométrie des sous-fibrés et des feuilletages lagrangiens, (French) [On the geometry of subbundles and Lagrange foliations], Ann. Sci. École Norm. Sup. (4) 14 (1981), no. 4, 465-480 (1982).

[ 8 ] M. Goto and F. D. Grosshans, Semisimple Lie Algebras, Lecture Notes in Pure and Applied Mathematics, Vol. 38. Marcel Dekker, Inc., New York-Basel, 1978.

[ 9 ] H. B. LAwSON, JR. AND J. Simons, On stable currents and their application to global problems in real and complex geometry, Ann. of Math. 98 (1973), 427-450.

[10] A. LiCHnEROwiCZ, Geometrie des groupes de transformations, Travaux et Recherches Mathematiques, III. Dunod, Paris, 1958

[11] O. Loos, Symmetric Spaces. I: General Theory, II: Compact Spaces and Classification, W. A. Benjamin, Inc., New York-Amsterdam, 1969.

[12] Y. Matsushima, Remarks on Kähler-Einstein manifolds, Nagoya Math. J. 46 (1972), 161-173.

[13] H. NAITOH, Totally real parallel submanifolds in $P^{n}(c)$, Tokyo J. Math. 4 (1981), 279-306.

[14] H. NAITOH, Parallel submanifolds of complex space forms. I, Nagoya Math. J. 90 (1983), 85-117.

[15] H. NAITOH, Parallel submanifolds of complex space forms. II, Nagoya Math. J. 91 (1983), 119-149.

[16] H. NAITOH AND M. TAKEUCHI, Totally real submanifolds and symmetric bounded domains, Osaka Math. J. 19 (1982), 717-731.

[17] K. OgIUE, Some recent topics in the theory of submanifolds, Sugaku Expositions 4 (1991), 21-41.

[18] Y. G. OH, Second variation and stabilities of minimal Lagrangian submanifolds in Kähler manifolds, Invent. Math. 101 (1990), 501-519.

[19] Y. G. OH, Tight Lagrangian submanifolds in $C P^{n}$, Math. Z. 207 (1991), 409-416.

[20] Y. G. Oh, Volume minimization of Lagrangian submanifolds under Hamiltonian deformations, Math. Z. 212 (1993), 175-192.

[21] Y. G. OH, Mean curvature vector and symplectic topology of Lagrangian submanifolds in Einstein-Kähler manifolds, Math. Z. 216 (1994), 471-482.

[22] Y. OHNiTA, Stable minimal submanifolds in compact rank one symmetric spaces, Tohoku Math. J. 38 (1986), 199-217. 
[23] H. OnO, Minimal Lagrangian submanifolds in adjoint orbits and upper bounds on the first eigenvalue of the Laplacian, J. Math. Soc. Japan 55 (2003), 243-254.

[24] T. TAKAHASHI, Minimal immersions of Riemannian manifolds, J. Math. Soc. Japan 18 (1966), 380-385.

[25] M. TAKeUCHI, Stability of certain minimal submanifolds of compact Hermitian symmetric space, Tohoku Math. J. 36 (1984), 293-314.

[26] M. TAKEUCHI, Modern Theory of Spherical Functions, Iwanami, Tokyo, 1975, (in Japanese). Modern Spherical Functions, Translations of Mathematical Monographs, 135. American Mathematical Society, Providence, RI, 1994.

[27] M. Takeuchi and S. Kobayashi, Minimal imbeddings of $R$-spaces, J. Differential Geom. 2 (1968), 203215.

[28] F. URBANO, Index of Lagrangian submanifolds of $\boldsymbol{C} P^{n}$ and the Laplacian of 1-forms, Geom. Dedicata 48 (1993), 309-318.

SCHOOL OF MATHEMATICS AND COMPUTER SCIENCE

NATIONAL UNIVERSITY OF MONGOLIA

UNIVERSITY STREET 3

P. O. BOX 46/145

VLAANBAATAR

MONGOLIA

E-mail address: zaya_math@yahoo.com
DEPARTMENT OF MATHEMATICS

GRAdUATE SCHOOL OF SCIENCE

TOKYO METROPOLITAN UNIVERSITY

MinAMI-OHSAWA 1-1, HACHIOJI

TOKYO 192-0397

JAPAN

E-mail address: ohnita@comp.metro-u.ac.jp 